\title{
SEPARATING BARYONS AND DARK MATTER IN CLUSTER CORES: A FULL TWO-DIMENSIONAL LENSING AND DYNAMIC ANALYSIS OF ABELL 383 AND MS 2137-23
}

\author{
David J. Sand, ${ }^{1,2}$ Tommaso Treu, ${ }^{3,4}$ Richard S. Ellis, ${ }^{5}$ Graham P. Smith, ${ }^{6}$ and Jean-Paul Kneib ${ }^{7}$ \\ Received 2007 March 9; accepted 2007 October 3
}

\begin{abstract}
We utilize existing data for the galaxy clusters MS 2137-23 and Abell 383 to present improved measures of the distribution of dark and baryonic material in the clusters' central regions. Our method, based on the combination of gravitational lensing and dynamical data, is uniquely capable of separating the distribution of dark and baryonic components at scales below $100 \mathrm{kpc}$. Our mass models include pseudoelliptical generalized NFW profiles for constraining the inner dark matter slope. We find that a variety of strong-lensing models fit the available data, including some with dark matter profiles as steep as expected from recent simulations. However, when combined with stellar velocity dispersion data for the brightest member, shallower inner slopes than predicted by numerical simulations are preferred, in general agreement with our earlier work in these clusters. For Abell 383, the preferred shallow inner slopes are statistically a good fit only when the multiple-image position uncertainties associated with our lens model are assumed to be $0.5^{\prime \prime}$, to account for unknown substructure. No statistically satisfactory fit was obtained matching both the multiple-image lensing data and the velocity dispersion profile of the brightest cluster galaxy in MS 2137-23. This suggests that the mass model we are using, which comprises a pseudoelliptical generalized NFW profile and a brightest cluster galaxy component, may inadequately represent the inner cluster regions. This may arise due to halo triaxiality or by the gravitational interaction of baryons and dark matter in cluster cores. The progress made via this detailed study highlights the key role that complementary observations of lensed features and stellar dynamics offer in understanding the interaction between dark and baryonic matter on nonlinear scales in the central regions of clusters.
\end{abstract}

Subject headings: dark matter — galaxies: formation — gravitational lensing

Online material: color figures

\section{INTRODUCTION}

Cold dark matter (CDM) simulations (both with and without the inclusion of baryonic physics) are a crucial tool and proving ground for understanding the physics of the universe on nonlinear scales. One of the most active aspects of research in this area concerns the form of the dark matter density profile. Key questions raised in recent years include the following: Is there a universal dark matter density profile that spans a wide range of halo masses? What is the form of this profile, and how uniform is it from one halo to another? To what extent do baryons modify the dark matter distribution?

Drawing on a suite of $N$-body simulations, Navarro et al. (1997) originally proposed that the dark matter density profiles in halos ranging in size from those hosting dwarf galaxies to those with galaxy clusters have a universal form. This three-dimensional (3D) density distribution, termed the "NFW profile," follows $\rho_{\mathrm{DM}} \propto r^{-1}$ within some scale radius, $r_{\mathrm{sc}}$, and falls off as $\rho_{\mathrm{DM}} \propto$ $r^{-3}$ beyond. Subsequent simulations indicated that the inner density profile could be yet steeper: $\rho_{\mathrm{DM}} \propto r^{-1.5}$ (Moore et al. 1998; Ghigna et al. 2000). As computing power increases and numerical techniques improve, it is now unclear whether the inner dark matter distribution converges to a power law form

\footnotetext{
1 Chandra Fellow.

2 Steward Observatory, University of Arizona, Tucson, AZ 85721.

3 Department of Physics, University of California, Santa Barbara, CA 93106-9530.

4 Alfred P. Sloan Research Fellow. 91125 .

5 Department of Astronomy, California Institute of Technology, Pasadena, CA

6 School of Physics and Astronomy, University of Birmingham, Edgbason, Birmingham B15 2TT, UK

7 OAMP, Laboratoire d'Astrophysique de Marseille, Traverse du siphon, 13012 Marseille, France.
}

rather than becoming progressively shallower in slope at smaller radii (Power et al. 2003; Navarro et al. 2004; Diemand et al. 2004, 2005).

For comparisons with data, such simulations need to account for the presence of baryons. This is particularly the case in the cores of rich clusters. Although baryons represent only a small fraction of the overall cluster mass, they may be crucially important on scales comparable to the extent of typical brightest cluster galaxies. Much work is being done to understand the likely interactions between baryons and DM (Gnedin et al. 2004; Nagai \& Kravtsov 2005; Faltenbacher et al. 2005). These simulations will provide refined predictions of the relative distributions of baryons and DM.

This paper is a further step in a series that aims to present an observational analog to progress described above in the numerical simulations. At each stage it is desirable to confront numerical predictions with observations. Whereas some workers have made good progress in constraining the total density profile (e.g., Broadhurst et al. 2005a), in order to address the relevance of the numerical simulations we consider it important to develop and test techniques capable of separating the distributions of dark and baryonic components (e.g., Sand et al. 2002; Zappacosta et al. 2006; Biviano \& Salucci 2006; Mahdavi et al. 2007).

This paper presents a refined version of the method first proposed by Sand et al. (2002) and exploited more fully in Sand et al. (2004, hereafter S04). S04 sought to combine constraints from the velocity dispersion profile of a central brightest cluster galaxy (BCG) with a strong gravitational lensing analysis in six carefully selected galaxy clusters in order to separate the baryonic and dark matter distributions. S04 carefully selected clusters to have simple, apparently "relaxed" gravitational potentials in order to match broadly the "equilibrium" status of the simulated dark matter halos originally analyzed by Navarro et al. (1997) and subsequent 
simulators. For example, Abell 383 and MS 2137-23 have almost circular BCGs $(b / a=0.90$ and 0.83 , respectively), require a single cluster dark matter halo to fit the strong-lensing constraints (in contrast to the more typical clusters that require a multimodal dark matter morphology; Smith et al. 2005), have previously published lens models with a relatively round dark matter halo $(b / a=0.88$ and 0.78 , respectively; Smith et al. 2001; Gavazzi 2005), and display no evidence for dynamical disturbance in the X-ray morphology of the clusters (Smith et al. 2005; Schmidt \& Allen 2007).

The merit of the approach resides in combining two techniques that collectively probe scales from the inner $\sim 10 \mathrm{kpc}$ (using the BCG kinematics) to the $\sim 100 \mathrm{kpc}$ scales typical of strong lensing. Whereas three of the clusters contained tangential arcs, constraining the total enclosed mass within the Einstein radius, three contained both radial and tangential gravitational arcs, the former providing additional constraints on the derivative of the total mass profile. In their analysis, S04 found that the gradient of the inner dark matter density distribution varied considerably from cluster to cluster, with a mean value substantially flatter than that predicted in the numerical simulations.

S04 adopted a number of assumptions in their analysis whose effect on the derived mass profiles was discussed at the time. The most important of these included ignoring cluster substructure and adopting spherically symmetric mass distributions centered on the BCG. The simplifying assumptions were considered sources of systematic uncertainties, of order 0.2 on the inner slope. Although the six clusters studied by $\mathrm{S} 04$ were carefully chosen to be smooth and round, several workers attributed the discrepancy between the final results and those of the simulations as likely to arise from these simplifying assumptions (Bartelmann \& Meneghetti 2004; Dalal \& Keeton 2003; Meneghetti et al. 2007).

The goal of this paper is to refine the data analysis for two of the clusters (MS 2137-23 and Abell 383) originally introduced by S04 using fully two-dimensional (2D) strong gravitational lensing models, thus avoiding any assumptions about substructure or spherical symmetry. The lensing models are based on an improved version of the LENSTOOL ${ }^{8}$ program (Kneib 1993; Kneib et al.1996; see the Appendix). A major development is the implementation, in the code, of a pseudoelliptical parameterization for the NFW mass profile, i.e., a generalization of those seen in CDM simulations, viz.,

$$
\rho_{d}(r)=\frac{\rho_{c} \delta_{c}}{\left(r / r_{\mathrm{sc}}\right)^{\beta}\left[1+\left(r / r_{\mathrm{sc}}\right)\right]^{3-\beta}},
$$

where the asymptotic DM inner slope is $\beta$. This formalism allows us to overcome an important limitation of previous work and takes into account the ellipticity of the DM halo and the presence of galaxy-scale subhalos. Furthermore, the 2D lensing model fully exploits the numerous multiply-imaged lensing constraints available for MS 2137-23 and Abell 383.

The combination of gravitational lensing and stellar dynamics is the most powerful way to separate baryons and dark matter in the inner regions of clusters. However, it is important to keep a few caveats in mind. Galaxy clusters are structurally heterogeneous objects that are possibly not well represented by simple parameterized mass models. To gain a full picture of their mass distribution and the relative contribution of their major mass components will ultimately require a variety of measurements applied simultaneously across a range of radii. Steps in this direction are already being made with the combined use of strong and weak

\footnotetext{
${ }^{8}$ See http://www.oamp.fr/cosmology/lenstool/.
}

gravitational lensing (e.g., Limousin et al. 2007; Bradač et al. 2006), which may be able to benefit further from information provided from X-ray analyses (e.g., Schmidt \& Allen 2007) and kinematic studies (e.g., Łokas \& Mamon 2003). A recent analysis has synthesized weak-lensing, X-ray, and Sunyaev-Zel'dovich observations in the cluster Abell 478; similar cross disciplinary work will lend further insights into the mass distribution of clusters (Mahdavi et al. 2007).

Of equal importance are mass models with an appropriate amount of flexibility and sophistication. For instance, incorporating models that take into account the interaction of baryons and dark matter may shed light into the halo formation process and provide more accurate representations of dark matter structure. Halo triaxiality, multiple structures along the line of sight, and other geometric effects will also be important to characterize. At the moment, incorporating these complexities and securing good parameter estimates is computationally expensive even with sophisticated techniques such as the Markov chain Monte Carlo method.

Numerical simulation results are often presented as the average profile found in the suite of calculations performed. Instead, the distribution of inner slopes would be a more useful quantity for comparison with individual cluster observations. Also, comparisons between simulations and observations would be simplified if projected density profiles of simulated halos along multiple lines of sight were to be made available. These issues should be resolvable as large samples of observed mass profiles are obtained.

For the reasons above, comparing observational results with numerical simulations is nontrivial. The observational task should be regarded as one of developing mass modeling techniques of increasing sophistication that separate dark and baryonic matter, so as to provide the most stringent constraints to high-resolution simulations that include baryons as they also increase in sophistication. The combination of stellar dynamics and strong lensing is the first crucial step in this process. Its diagnostic power will be further enhanced by including other major mass components (i.e., the hot gas of the intracluster medium or the stellar contribution from galaxies) out to larger radii.

A plan of the paper follows. In $\S 2$ we explain the methodology used to model the cluster density profile by combining strong lensing with the BCG velocity dispersion profile. In $\S 3$ we focus on translating observational measurements into strong-lensing input parameters. This section includes the final strong-lensing interpretation of MS 2137-23 and Abell 383. In $\S 4$ we present the results of our combined lensing and dynamical analysis. In $\S 5$ we discuss further systematic effects, limitations, and degeneracies that our technique is susceptible to, with an eye toward future refinements. Finally, in $\S 6$ we summarize and discuss our conclusions. Throughout this paper we adopt $r$ as the radial coordinate in $3 \mathrm{D}$ space and $R$ as the radial coordinate in $2 \mathrm{D}$ projected space. When necessary, we assume $H_{0}=65 \mathrm{~km} \mathrm{~s}^{-1} \mathrm{Mpc}^{-1}$, $\Omega_{m}=0.3$, and $\Omega_{\Lambda}=0.7$.

\section{METHODS}

The intent of this work is to use the full 2D information provided by the deep Hubble Space Telescope (HST) imaging in two strong-lensing clusters (MS 2137-23 and Abell 383) in conjunction with the BCG stellar velocity dispersion profile in order to constrain the distribution of baryonic and dark matter. These two clusters were selected for further study from the larger sample presented by S04 because, of the three systems with both radial and tangential gravitational arcs, these two presented the shallowest DM inner slopes. 
TABLE 1

Acceptable Parameter Range

\begin{tabular}{|c|c|c|c|c|c|c|}
\hline Prior Setup & Cluster & Inner DM Slope $\beta$ & $\epsilon$ & $\delta_{c}$ & $\begin{array}{c}r_{\mathrm{sc}} \\
(\mathrm{kpc})\end{array}$ & $M_{*} / L_{V}$ \\
\hline \multicolumn{7}{|c|}{$100-200 \mathrm{kpc} r_{\mathrm{sc}}$ Prior } \\
\hline \multirow[t]{2}{*}{$\sigma_{\text {lens }}=0.2^{\prime \prime} \ldots \ldots \ldots \ldots \ldots \ldots$} & MS 2137 & $0.95_{-0.30}^{+0.05}$ & $0.08_{-0.01}^{+0.01}$ & $29420_{-1760}^{+98310}$ & $200^{-42}$ & $1.58_{-0.635}^{+0.52}$ \\
\hline & Abell 383 & $0.55_{-0.05}^{+0.20}$ & $0.08_{-0.02}^{+0.01}$ & $140000_{-60600}^{+8500}$ & $100^{+28}$ & $2.4_{-0.42}^{+0.42}$ \\
\hline \multirow{2}{*}{$\sigma_{\text {lens }}=0.5^{\prime \prime} \ldots \ldots \ldots \ldots \ldots$} & MS 2137 & $0.6^{+0.15}$ & $0.06^{-0.01}$ & $44600^{-60600}+3500$ & $200^{-31}$ & $2.45^{-0.45}$ \\
\hline & Abell 383 & $0.45_{-0.25}^{+0.2}$ & $0.06_{-0.01}^{+0.01}$ & $156000_{-67150}^{+38500}$ & $100^{+21}$ & $2.34_{-0.54}^{+1.03}$ \\
\hline
\end{tabular}

NotE.-Best-fitting parameters and/or confidence limits for the different prior scenarios present in this paper.

\subsection{Lens Modeling}

We use the updated LENSTOOL ray-tracing code to construct models of the cluster mass distribution. Our implementation of the mass profiles is identical to that of Golse \& Kneib (2002) with the exception that we have generalized their pseudoelliptical parameterization to include ones with arbitrary inner logarithmic slopes. For the details, the reader is referred to both Golse $\&$ Kneib (2002) and the Appendix. Here we briefly explain the lens modeling process and parameterization of the cluster mass model.

Identifying mass model components and multiple-image candidates is an iterative process. Initially, multiple images are spectroscopically confirmed systems with counterimages identified by visual inspection and with the aid of preliminary lens models, taking into account that gravitational lensing conserves surface brightness. Multiple images without spectroscopic confirmation were used in the case of Abell 383, since these additional constraints helped clarify the role that galaxy perturber 1 (Table 1) played in the central regions of the cluster (see $\S 3.3$ ). If the location of a counterimage is tentative, especially if there are several possibilities or an intervening cluster galaxy confuses the situation, the system is not included in deriving the mass model. In $\S \S 3.2$ and 3.3 we present a detailed description of the final multiple-image list adopted.

Once the multiple images are determined, the cluster mass model is refined and perturber galaxy properties are fixed. In general, a lens mass model will have both cluster- and galaxy-scale mass components. The cluster-scale mass component represents the DM associated with the cluster as a whole plus the hot gas in the intracluster medium. In the limit that the cluster DM halo is spherical (see eq. [A6]), its density profile has the form of equation (1). In the adopted parameterization, the DM halo also has a position angle $(\theta)$ and associated pseudoellipticity $(\epsilon)$ (see eqs. [A5] and [A6]).

Galaxy-scale mass components are necessary to account for perturbations to the cluster potential that seem plausible based on the HST imaging and are demanded by the observed multipleimage positions. These components are described by pseudoisothermal elliptical mass distributions (PIEMDs; Kassiola \& Kovner 1993). Each PIEMD mass component is parameterized by its position $\left(x_{c}, y_{c}\right)$, ellipticity $(e)$, position angle $(\theta)$, core radius $\left(r_{\text {core }}\right)$, cutoff radius $\left(r_{\text {cut }}\right)$, and central velocity dispersion $\left(\sigma_{0}\right)$. The projected mass density, $\Sigma$, is given by

$$
\Sigma(x, y)=\frac{\sigma_{0}^{2}}{2 G} \frac{r_{\text {cut }}}{r_{\text {cut }}-r_{\text {core }}}\left[\frac{1}{\left(r_{\text {core }}^{2}+\rho^{2}\right)^{1 / 2}}-\frac{1}{\left(r_{\text {cut }}^{2}+\rho^{2}\right)^{1 / 2}}\right],
$$

where $\rho^{2}=\left[\left(x-x_{c}\right) /(1+e)\right]^{2}+\left[\left(y-y_{c}\right) /(1-e)\right]^{2}$ and the ellipticity of the lens is defined as $e=(a-b) /(a+b))^{9}$ The total mass of the PIEMD is thus $3 / 2 \pi \sigma_{0}^{2} r_{\text {cut }} / G$. In order to relate equation (2) to the observed surface brightness of the BCG in particular, we take $\Sigma=\left(M_{*} / L\right) I$, where $M_{*} / L$ is the stellar mass-to-light ratio and $I$ is the surface brightness, and find the following relation:

$$
M_{*} / L=1.50 \pi \sigma_{0}^{2} r_{\text {cut }} /(G L)
$$

where $L$ is the total luminosity of the BCG. The $M_{*} / L$ of the central BCG will be used as a free parameter in our mass modeling analysis. Further details and properties of the truncated PIEMD model can be found in Natarajan \& Kneib (1997) and Limousin et al. (2005).

The relevant parameters of the perturber galaxies (position, ellipticity, core radius, cutoff radius, and position angle) are assumed to be those provided via examination of the HST imaging (see $\S 3.1$ for details). Only the central stellar velocity dispersion, $\sigma_{0}$, is determined by optimization. At a particular stage in the process, the predicted multiple-image positions are compared with those observed, and a $\chi^{2}$ value calculated (see $\S 2.3$ ). The iteration stops when a $\chi^{2}$ minimum is reached. The sole criterion for adding a perturber galaxy was whether or not it was necessary for the lens model to match the multiple-image positions. If adding an additional perturber did not alter our interim minimum $\chi^{2}$, we did not include it in our subsequent analysis.

Two special cases were encountered during the above procedure. First, one of the galaxy perturbers in Abell 383 required a larger cutoff radius $\left(r_{\text {cut }}\right)$ than implied from the light distribution. As is described in $\S 3.3$, this concentration is necessary to account for several of the multiple-image positions. For this mass concentration we determine not only the optimum $\sigma_{0}$ parameter but also the cutoff radius $\left(r_{\text {cut }}\right)$.

The other special case concerns the BCG in both galaxy clusters. These are assumed to be coincident with the center of the cluster DM halo, justified by the colocation of the BCG and central X-ray isophotes (Smith et al. 2005; Gavazzi et al. 2003). The BCG mass distribution, represented by a PIEMD model, comprises only the stellar mass. In this case the HST imaging is used to fix the BCG ellipticity and position angle, but since the measured stellar velocity dispersion is to be used as a constraint on the cluster mass profile, we leave the central velocity dispersion parameter (and hence the stellar $M_{*} / L$; eq. [3]) free in the lensing analysis. As the Jaffe density profile is used for the BCG dynamical analysis (S04), the PIEMD core and cutoff radius that best match the Jaffe surface density are adopted. Section 3.1 discusses further

\footnotetext{
9 This quantity should not be confused with the quite different definition used for the pseudoelliptical gNFW profile; see the Appendix.
} 
the results of the surface brightness matching between the Jaffe and PIEMD models in the clusters.

\subsection{Incorporating the Dynamical Constraints}

Apart from the use of the pseudoelliptical generalized NFW (gNFW) profile for the dark matter component, the observational data and analysis methods adopted here are identical to those used by S04. In that work, the observed velocity dispersion profile of the BCG was interpreted via the spherical Jeans equation (see Appendix of S04) to assist in the decomposition of the dark and baryonic mass components. This portion of the $\chi^{2}$ was calculated by comparing the expected velocity dispersion profile of the BCG (which depends on the mass enclosed at a given radius and the relative contribution of dark and luminous matter) given a mass model with the observed velocity dispersion profile, taking into account the effects of seeing and the long-slit shape used for the observations. Ellipticity in the BCG and its dark matter halo can be ignored as its effect on the velocity dispersion profile will be small (e.g., Kronawitter et al. 2000).

The reader is referred to S04 for the observational details pertaining to the velocity dispersion profile, the surface brightness profile of the BCG, and the subsequent dynamical modeling to constrain the cluster DM inner slope.

\subsection{Statistical Methods}

A $\chi^{2}$ estimator is used to constrain the acceptable range of parameters compatible with the observational data. First we use the strong-lens model to calculate the likelihood of the lensing constraints, and then we combine it with a dynamical model to include the kinematic information in the likelihood.

The first step is the strong-lensing likelihood. Once the lensing interpretation is finalized and the perturbing galaxy parameters are fixed, the remaining free parameters are constrained by calculating a lensing $\chi^{2}$ over a hypercube that encompasses the full range of acceptable models, modulo a prior placed on the dark matter scale radius (see $\S 2.4$ ). In Bayesian terms this corresponds to adopting a uniform prior. The lensing $\chi^{2}$ value is calculated in the source plane identically to that of Smith et al. (2005). For each multiply-imaged system, the source location for each noted image $\left(x_{\text {model }, i}, y_{\text {model }, i}\right)$ is calculated using the lens equation. Since there should be only one source for each multipleimage set (with $N$ images), the difference between the source positions should be minimized, hence

$$
\chi_{\text {pos }}^{2}=\sum_{i=1}^{N} \frac{\left(x_{\text {model }, i}-x_{\text {model }, i+1}\right)^{2}+\left(y_{\text {model }, i}-y_{\text {model }, i+1}\right)^{2}}{\sigma_{S}^{2}} .
$$

Parameter $\sigma_{S}$ is calculated by scaling the positional error associated with a multiple-image knot, $\sigma_{I}$, by the amplification of the source $A$ so that $\sigma_{I}^{2}=A \sigma_{S}^{2}$. The following analysis assumes two different positional errors for the multiply-imaged knots, using uncertainties of $\sigma_{I}=0.2^{\prime \prime}$ and $0.5^{\prime \prime}$, referred to hereafter as the "fine" and "coarse" analyses, respectively. The case for each is justified below.

The finer $0.2^{\prime \prime}$ error bar corresponds to the uncertainty in the multiple-image knot positions as defined by the resolution and pixel size of the HST WFPC2 images. Excellent strong-lensing fits are achieved with the finer $0.2^{\prime \prime}$ error bar $\left(\chi^{2} /\right.$ dof $\left.\sim 1\right)$, so that technically there is no need for increased uncertainties. The uncertainty is dominated by the spatial extension of the multiple- image knots employed and the ability to identify surface brightness peaks.

In contrast to our ability to match the image positions down to the resolution of the HST WFPC2 images, recent combined strongand weak-lensing analyses of Abell 1689 have been unable to do so (e.g., Broadhurst et al. 2005b; Halkola et al. 2006; Limousin et al. 2007). Although Abell 1689 is a more complex cluster than those studied here, it does have the most identified multiple images of any other cluster to date and so can probe the overall mass profile on smaller scales and with many more constraints. As espoused in $\S 1$, real galaxy clusters are complex systems that are likely not easily parameterized by simple mass models, and as the number and density of mass probes increase, the more refined and complete the mass model necessary to match the data. In our case, where we have relatively few mass profile constraints (at least in comparison to Abell 1689), we adopt a coarser $0.5^{\prime \prime}$ positional error that allows us to account for complexities in the actual mass distribution of our clusters that our small number of mass probes are insensitive to. This, plus the fact that we carefully chose our perturbing galaxies such that a lensing $\chi^{2} /$ dof $\sim 1$ was found, should account for reasonable situations where we have missed an interesting perturber galaxy. By adopting too small a multipleimage position uncertainty, the region in parameter space explored may be overly confined (such that, for example, the observed BCG velocity dispersion profile cannot be reproduced).

The strong-lensing analysis is performed with five free parameters, analogous to those adopted in S04. These are the dark matter inner logarithmic slope $(\beta)$, the pseudoellipticity of the potential $(\epsilon)$, the amplitude of the DM halo $\left(\delta_{c}\right)$, the dark matter scale radius $\left(r_{\mathrm{sc}}\right)$, and the mass-to-light ratio of the BCG $\left(M_{*} / L\right)$. We choose to place a uniform prior on the dark matter scale radius $\left(r_{\mathrm{sc}}\right)$ based on past mass profile analyses of these clusters and results from CDM simulations in order to reduce computation time (see $\S 2.4$ ). In practice, to evaluate the $\chi_{\text {pos }}^{2}$ at each point in the hypercube, the pseudoellipticity of the cluster dark matter halo is optimized while simply looping over the remaining free parameters.

Once the strong-lensing $\chi^{2}$ values are computed, attention is turned to the dynamical data. In contrast to the strong-lensing model, the dynamical model is spherically symmetric and follows that presented by S04, with the $\chi^{2}$ value being

$$
\chi_{\sigma}^{2}=\sum_{i=1}^{N} \frac{\left(\sigma_{i, \mathrm{obs}}-\sigma_{i, \mathrm{model}}\right)^{2}}{\Delta_{i}^{2}},
$$

where $\Delta_{i}$ is the uncertainty in the observed velocity dispersion measurements.

The lensing and velocity dispersion $\chi^{2}$ values are summed, allowing for standard marginalization of nuisance parameters and the calculation of confidence regions.

\subsection{Dark Matter Scale Radius Prior}

As mentioned in the previous sections, in order to limit computation time, a prior was placed on the dark matter scale radius $r_{\text {sc }}$. This is justified on both previous mass profile analyses of these clusters and the results of CDM simulations.

An array of CDM simulations has provided information not only on the inner dark matter density profile but also on the expected value of the scale radius, $r_{\mathrm{sc}}$, and its intrinsic scatter at the galaxy cluster scale (e.g., Bullock et al. 2001; Tasitsiomi et al. 2004; Dolag et al. 2004). For example, Bullock et al. (2001) found that dark matter halos the size of small galaxy clusters have scale radii between 240 and $550 \mathrm{kpc}(68 \% \mathrm{CL})$. Tasitsiomi et al. (2004), using higher resolution simulations with fewer dark matter halos, found 
TABLE 2

Fixed Parameters in Abell 383 and MS 2137-23 Lens Model

\begin{tabular}{|c|c|c|c|c|c|c|c|c|}
\hline Cluster & Parameters & $\begin{array}{c}x_{c} \\
(\operatorname{arcsec})\end{array}$ & $\begin{array}{c}y_{c} \\
(\operatorname{arcsec})\end{array}$ & $b / a$ & $\begin{array}{c}\theta \\
(\mathrm{deg})\end{array}$ & $\begin{array}{c}r_{\text {core }} \\
(\mathrm{kpc})\end{array}$ & $\begin{array}{c}\sigma_{0} \\
\left(\mathrm{~km} \mathrm{~s}^{-1}\right)\end{array}$ & $\begin{array}{r}r_{\mathrm{cut}} / R_{e} \\
(\mathrm{kpc})\end{array}$ \\
\hline \multirow[t]{3}{*}{ MS 2137} & Cluster-scale DM halo & 0.0 & 0.0 & Free & 5.0 & $\ldots$ & ... & $\ldots$ \\
\hline & $\mathrm{BCG}_{\mathrm{Jaffe}}$ & 0.0 & 0.0 & $\ldots$ & $\ldots$ & $\ldots$ & $\ldots$ & 24.80 \\
\hline & Galaxy perturber & 16.2 & -5.46 & 0.66 & 159.9 & 0.05 & 173.0 & 4.8 \\
\hline \multirow[t]{5}{*}{ Abell 383 ............................... } & Cluster-scale DM halo & 0.0 & 0.0 & Free & 104.3 & $\ldots$ & $\ldots$ & $\ldots$ \\
\hline & $\mathrm{BCG}_{\text {PIEMD }}$ & 0.0 & 0.0 & 0.90 & 107.2 & $3 \times 10^{-6}$ & Free & 25.96 \\
\hline & Perturber 2 & 9.15 & -1.92 & 0.708 & 10.3 & 0.51 & 140.0 & 10.79 \\
\hline & Perturber 3 & 0.17 & -24.26 & 0.67 & 65.2 & 0.24 & 124.8 & 9.10 \\
\hline & Perturber 4 & -4.10 & -13.46 & 0.645 & 27.7 & 0.17 & 125.7 & 2.19 \\
\hline
\end{tabular}

Notes.-The position angle, $\theta$, is measured from north toward east. The DM halo is parameterized with the pseudo-gNFW profile. All other mass components are parameterized by a PIEMD model. Note that $R_{e}=0.76 r_{\text {Jaffe }}$ (Jaffe 1983).

$r_{\mathrm{sc}}$ of $450 \pm 300 \mathrm{kpc}$. Finally, Dolag et al. (2004) studied the DM concentrations of galaxy clusters in a $\Lambda \mathrm{CDM}$ cosmology and found a typical range of scale radii $r_{\mathrm{sc}}$ between 150 and $400 \mathrm{kpc}$. These results represent a selection of the extensive numerical work being done on the concentration of dark matter halos.

Previous combined strong- and weak-lensing analyses of MS 2137-23 have provided approximate values for the scale radius (Gavazzi et al. 2003; Gavazzi 2005). Gavazzi et al. (2003) found a best-fitting scale radius of $\sim 130 \mathrm{kpc}$ (and hints that the scale radius may be as low as $\sim 70 \mathrm{kpc}$ from their weak-lensing data) for their analysis of MS 2137-23. A more recent analysis (Gavazzi 2005) found a best-fitting radius of $\sim 170 \mathrm{kpc}$. Similarly for Abell 383, a recent X-ray analysis found a best-fitting dark matter scale radius of $\sim 130 \mathrm{kpc}$ (Zhang et al. 2007).

Taking these observational studies into account, we chose a uniform scale radius prior between 100 and $200 \mathrm{kpc}$ for MS 2137-23 and Abell 383, both for simplicity and to bracket the extant observational results which often have constraints at larger radii (and thus constrain the scale radius better) than the current work. It is worth noting that the extant observations of these two clusters indicate a scale radius that is on the low end of that predicted from CDM simulations. For a fixed virial mass, a smaller scale radius indicates a higher concentration, $c=r_{\mathrm{vir}} / r_{\mathrm{sc}}$. This could be due to the effect of baryonic cooling, which could increase halo concentration (as well as inner slope perhaps). It has been suggested that those halos with the highest concentration (again for a fixed mass) are those that are the oldest and with the least substructure, providing more indirect evidence that we have chosen relaxed galaxy clusters to study (Zentner et al. 2005). We briefly explore the consequences of changing our assumed scale radius prior range in $\S$ 5.2.2.

\section{APPLICATION TO DATA}

We now turn to the observational data for MS 2137-23 and Abell 383 and describe our methods for analyzing these in the context of lensing input parameters.

\subsection{BCG and Perturber Galaxy Parameters}

In order to fix the position angle and ellipticity of the perturber galaxies and BCG components, the IRAF task ellipse is used to estimate the surface brightness profile at typically the effective radius. The measured parameters are fixed in the lensing analysis. The galaxy position, core radius $\left(r_{\text {core }}\right)$, and cutoff radius $\left(r_{\text {cut }}\right)$ are each chosen to match those fitting the photometry (Table 1). For perturbing galaxies, this leaves only the PIEMD parameter velocity dispersion $\left(\sigma_{0}\right)$, which must be adjusted to match the multiple imaging constraints, as explained in $\S 2.1$.

For the BCG, following S04, it is preferable to use the Jaffe stellar density profile for the dynamical analysis since this function provides an analytic solution to the spherical Jeans equation. However, the PIEMD model implemented in LENSTOOL offers numerous advantages for the lensing analysis. To use the most advantageous model in each application, a correspondence is established between the two by fitting with a PIEMD model the Jaffe surface brightness fit presented by S04. An appropriate combination of the PIEMD $r_{\text {core }}$ and $r_{\text {cut }}$ model parameters matches the Jaffe profile found by S04 with no significant residuals (PSF smearing was also taken into account). Table 2 lists the PIEMD parameters used for our lensing analysis, as well as the Jaffe profile parameters used by S04.

\subsection{MS 2137-23 Lens Model}

The strong-lensing properties of MS 2137-23 have been studied extensively by many workers (Mellier et al. 1993; MiraldaEscude 1995; Hammer et al. 1997; Gavazzi et al. 2003; Gavazzi 2005). The most detailed model (Gavazzi et al. 2003) used 26 multiply-imaged knots from two different background sources. The model adopted here is more conservative and based only on those multiple images confirmed via spectroscopy or suggested on the grounds of surface brightness or interim lens models. Despite having some multiple images in common with Gavazzi et al. (2003), we have retained our own nomenclature.

Following Sand et al. (2002), the tangential and radial arcs arise from separate sources, at $z=1.501$ and 1.502 , respectively. The multiple-image interpretation is detailed in Figure 1 and Table 3. There are two separate features ( 1 and 3 ) on the source giving rise to the tangential arc, which is multiply imaged four and three times, respectively. It has not been possible to confidently locate the fourth image of feature 3 , since it is adjacent to the perturbing galaxy. Also, it is expected that a fifth, central image would be associated with the giant tangential arc. Although the position of this fifth central image has been tentatively reported (Gavazzi et al. 2003), we do not include it in our model because we were unable to clearly identify it due to BCG subtraction residuals. Two images of the source giving rise to the radial arc were also identified. The mirror image of feature 2a nearer the center of the BCG could not 

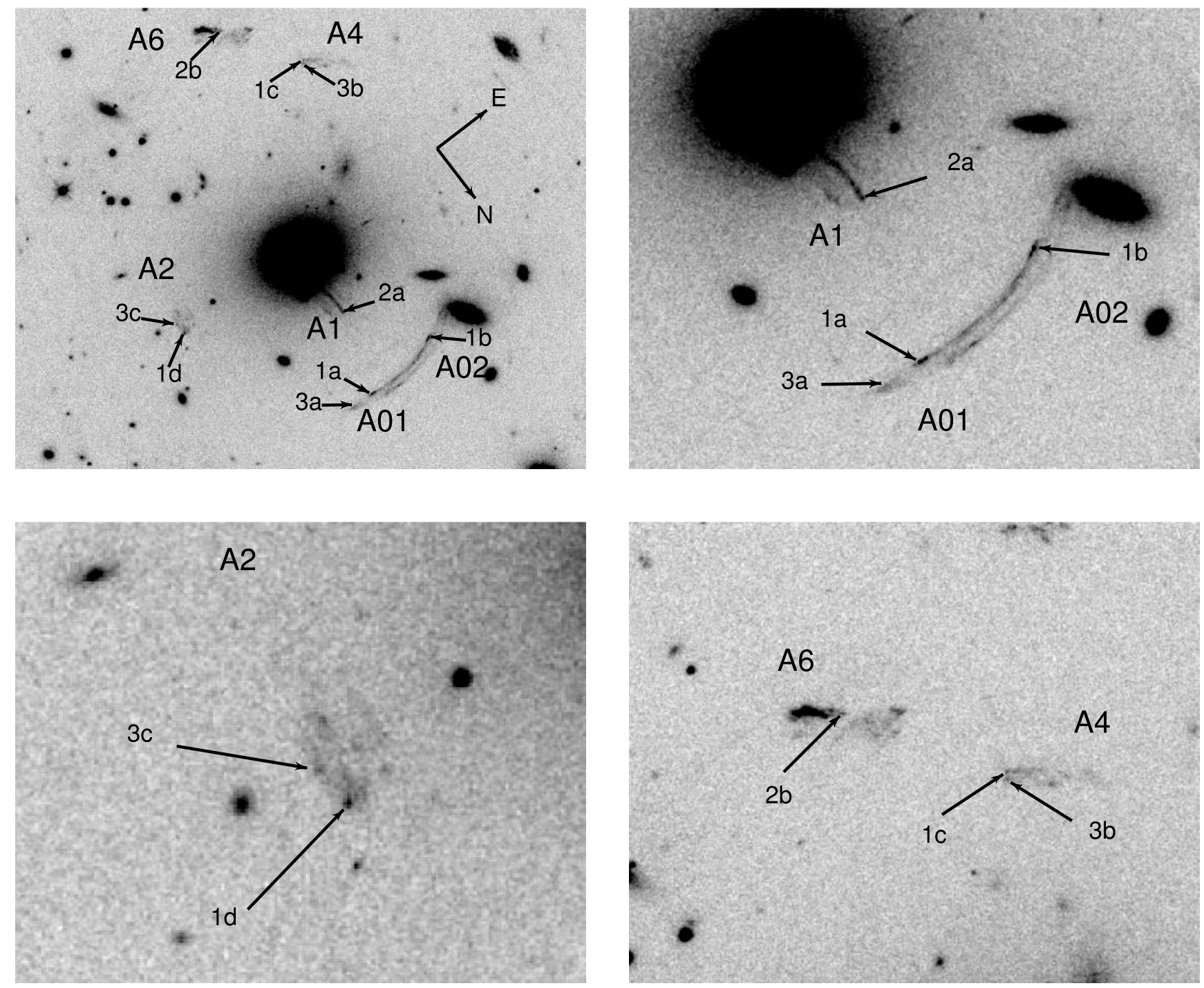

FIG. 1.-Multiple-image interpretation of the cluster MS 2137-23. The exact positions used are shown in Table 3. Three sets of multiple images are identified, one with the radial arc system ( $2 \mathrm{a}$ and $2 \mathrm{~b}$ ) and two with the tangential arc system (1abcd and 3abc). The perturbing galaxy is the elliptical S0 next to the lensed feature $1 \mathrm{~b}$.

be recovered, most likely because of residuals arising from the subtraction of the BCG.

As in Gavazzi et al. (2003), only one perturbing galaxy is included in the lens model (see Table 2). For this system, the bestfitting $\chi^{2}$ value occurred near $\sigma_{0}=173 \mathrm{~km} \mathrm{~s}^{-1}$.

In the initial modeling of MS 2137-23, some experimentation was undertaken with different cluster DM ellipticities and position angles. While some variation in ellipticity is permitted by the lensing interpretation, a robust position angle offset was detected between the BCG and that of the DM halo of $\Delta \theta \sim 13^{\circ}$, in agreement with Gavazzi et al. (2003). In the following, results are presented with the DM position angle fixed at $\theta=5^{\circ}$. This optimal position angle was determined during the initial lens modeling process by fixing all cluster mass parameters to values corresponding to a model with $\chi^{2}$ /dof $\sim 1$ and letting the DM position angle vary until a $\chi^{2}$ minimum was reached. As a consistency check, we repeated our calculations with a fixed DM position angle of $4.0^{\circ}$ and $6.0^{\circ}$. Varying the DM position angle had very little effect on our other parameter constraints but results in a slightly larger overall $\chi^{2}$ (lensing+velocity dispersion profile; $\left.\Delta \chi^{2}<1\right)$ value. For this reason, we only present our results with a DM position angle of $5^{\circ}$.

\subsection{Abell 383 Lens Model}

Detailed lens models for Abell 383 have been published in Smith et al. (2001, 2005), which we largely adopt in this work. Multiple-image sets 1 and 2 are based on the in-depth lensing interpretation of Smith et al. (2005). The reader is referred to that work for a detailed description of this radial and tangential gravitational arc. Multiple-image sets 3, 4, 5, and 6 (for which there are no spectroscopic redshifts, but for which their distinctive morphology is reassuring) are included largely to constrain the properties of perturbing galaxies 1, 3, and 4 (see Fig. 2 and Table 3). Since these images have no spectroscopic confirmation, a redshift $z \sim 3$ was assumed; the mass model is very insensitive to the exact choice.

The Abell 383 cluster mass model is more complex than that for MS 2137-23, but only in the sense that there are more perturbing galaxies that must be put into the mass model to match the image positions. The bright cluster elliptical southwest of the BCG ( 2 in Smith et al. 2005; perturber 1 in this work) requires a DM halo more extended than the light, as mentioned in $\S 2$. . After some iteration, it was found that the parameters of this important perturber could be fixed to those listed in Table 2. Multiple-image 
TABLE 3

Multiple-Image Interpretation of MS 2137 and Abell 383

\begin{tabular}{|c|c|c|c|c|}
\hline Cluster & Multiple-Image ID & $\begin{array}{c}x_{c} \\
(\operatorname{arcsec})\end{array}$ & $\begin{array}{c}y_{c} \\
(\operatorname{arcsec})\end{array}$ & Redshift \\
\hline \multirow[t]{9}{*}{ 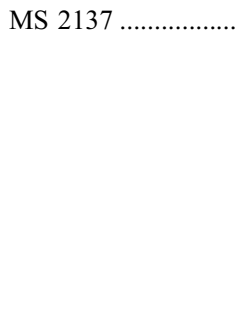 } & $1 \mathrm{a}$ & 6.92 & -13.40 & 1.501 \\
\hline & $1 b$ & 12.40 & -7.94 & 1.501 \\
\hline & $1 \mathrm{c}$ & 0.07 & 19.31 & 1.501 \\
\hline & $1 d$ & -11.57 & -7.49 & 1.501 \\
\hline & $2 \mathrm{a}$ & 3.96 & -5.51 & 1.502 \\
\hline & $2 b$ & -8.01 & 22.10 & 1.502 \\
\hline & $3 a$ & 5.16 & -14.68 & 1.501 \\
\hline & $3 b$ & 0.11 & 18.91 & 1.501 \\
\hline & $3 c$ & -12.30 & -6.74 & 1.501 \\
\hline \multirow[t]{16}{*}{ Abell 383 .................. } & $1 \mathrm{~A}$ & -1.74 & 2.56 & 1.0 \\
\hline & $1 \mathrm{~B}$ & -1.03 & 1.20 & 1.0 \\
\hline & $1 \mathrm{C}$ & 16.37 & -4.03 & 1.0 \\
\hline & $2 \mathrm{~A}$ & 7.00 & -14.01 & 1.0 \\
\hline & $2 \mathrm{~B}$ & 8.23 & -13.20 & 1.0 \\
\hline & $2 \mathrm{C}$ & 14.11 & -8.19 & 1.0 \\
\hline & $3 \mathrm{~A}$ & 5.88 & -22.02 & 3.0 \\
\hline & $3 \mathrm{~B}$ & 14.69 & -14.68 & 3.0 \\
\hline & $3 \mathrm{C}$ & 16.49 & -14.39 & 3.0 \\
\hline & $4 \mathrm{~A}$ & 8.35 & -23.96 & 3.0 \\
\hline & $4 \mathrm{~B}$ & 17.45 & -17.28 & 3.0 \\
\hline & $4 \mathrm{C}$ & 17.92 & -15.43 & 3.0 \\
\hline & $5 \mathrm{~A}$ & 6.64 & -21.75 & 3.0 \\
\hline & $5 \mathrm{~B}$ & 16.98 & -14.09 & 3.0 \\
\hline & $6 \mathrm{~A}$ & 7.05 & -21.75 & 3.0 \\
\hline & $6 \mathrm{~B}$ & 17.27 & -14.17 & 3.0 \\
\hline
\end{tabular}

NotE.-All image positions are with respect to the BCG center.

sets 3,4 , and 5 play a crucial role (see Table 3 ) in constraining the perturber. Although other perturbing galaxies were added, none have a comparable effect on the lensing $\chi^{2}$. Table 2 provides the full model parameter list.

A slight $\left(\sim 3^{\circ}\right)$ offset between the position angle of the BCG and the cluster DM halo was noted. We found the best-fitting DM position angle in the same way as in MS 2137-23 (§3.2). The position angle of the DM halo was kept fixed, but the ellipticity was left as a free parameter. As in MS 2137-23, we also reran our analysis with a DM position angle of 103.3 and 105.3 but only present the results with a DM position angle of 104.3.

\section{RESULTS}

We now analyze the refined 2D lens models of MS 2137-23 and Abell 383 together with the velocity dispersion profiles presented in S04. We present our analysis with a multiple-image position uncertainty of $0.2^{\prime \prime}$ and $0.5^{\prime \prime}$, described as the fine and coarse fits, respectively, in $\S 2.3$. The results are summarized in Table 1 and Figures 3 and 4.

\section{1. $M S$ 2137-23}

Figure 3 and the discussion below summarize the results for the fine and coarse positional cases. One thing to note is the number of degrees of freedom involved, i.e., the difference between the number of constraints and the number of free parameters, in order to quantify the goodness of fit. The mass model has five free parameters, as detailed in $\S 3.2$ : the DM inner slope $\beta$, the DM pseudoellipticity $\epsilon$, the DM amplitude $\delta_{c}$, the BCG stellar $M_{*} / L$, and the dark matter scale radius $r_{\mathrm{sc}}$, which is allowed to vary in the 100-200 kpc range. Considering Table 3, the multiple images provide 12 constraints, while the velocity dispersion data provide eight, giving a total of 20 data constraints. The resulting number of degrees of freedom is thus 15 .

\subsubsection{The Fine Positional Accuracy Lensing Case}

The two panels of Figure 3 and the appropriate line in Table 1 encapsulate the results of the fine positional analysis. DM inner slopes between $\beta=0.65$ and 1.0 lie within the $68 \%$ confidence limit (after marginalizing over all other free parameters), although the best-fitting DM scale radius sits at the edge of the allowed prior $\left(r_{\mathrm{sc} \text {,best }}=200 \mathrm{kpc}\right)$. A scale radius of $200 \mathrm{kpc}$ is higher than that seen in previous lensing analyses of MS 2137-23 that have used a similar mass parameterization to our own (e.g., Gavazzi et al. 2003; Gavazzi 2005), so there is no case to alter the prior.

The parameter constraints are not particularly tight because the total $\chi^{2} \sim 54$, larger than expected given the number of degrees of freedom. Such a value may indicate that the form of the mass profile used in the fit is inappropriate. Indeed, the model velocity dispersion profile is a poor match to that observed (Fig. 3). In fact, if the BCG velocity dispersion results are ignored, acceptable lens models $\left(\chi^{2} /\right.$ dof $\left.\lesssim 1\right)$ can be recovered with a variety of inner DM slopes, scale radii, and BCG stellar $M / L$, although these parameters have correlated values. We postpone discussion of the possible reasons for this mismatch until later.

\subsubsection{The Coarse Positional Accuracy Lensing Case}

The bottom panels of Figure 3, along with Table 1, summarize our results for the coarse positional analysis. DM inner slopes between $\beta=0.4$ and 0.75 lie within our $68 \% \mathrm{CL}$, and we again find DM scale radii at the high end of our prior range, as expected. The shift in the BCG $M / L$ versus DM inner slope contour fine positional case indicates that the increased parameter space in the lensing models has led to a slightly improved velocity dispersion profile (Fig. 3, bottom right panel). The overall $\chi^{2} \simeq 31$ is improved, although the probability for 15 degrees of freedom is less than $1 \%$, assuming that measurements are governed by Gaussian statistics. Thus, the model remains a marginal fit to the data.

\subsubsection{Comparison with Gavazzi (2005)}

We now briefly compare our results with those of Gavazzi (2005). Gavazzi's analysis used a similar strong-lensing model to our own, including what we consider to be somewhat less secure multiple images. However, he extended the analysis to larger scales including weak-lensing data and incorporated the BCG velocity dispersion profile presented by S04. Gavazzi adopted a strict NFW profile for the cluster DM halo and a Hernquist profile for the stellar component of the BCG.

Despite these differences, Gavazzi's conclusions are very similar to those of the present paper. Models with NFW-like DM halos (regardless of whether the inner slopes were varied) were uniformly poor fits to the observational data. In particular, the falling velocity dispersion profile observed at $R \gtrsim 5 \mathrm{kpc}$ cannot be reproduced, despite experimenting with the effect of anisotropic orbits in the stellar distribution. A major conclusion of Gavazzi's study is that halo triaxiality, an effect not typically included, may play an important role in the central regions of galaxy clusters. We return to this topic in $\S 5.2 .3$.

\subsection{Abell 383}

Our results for Abell 383 are shown in Figure 4 and Table 1 for both the fine and coarse positional uncertainty cases. We discuss each separately below. Calculating the number of degrees of freedom in a similar way to that done for MS 2137-23, we again have five free parameters in our mass model. Considering Table 3 , multiple images provide 16 constraints, taking into account that 

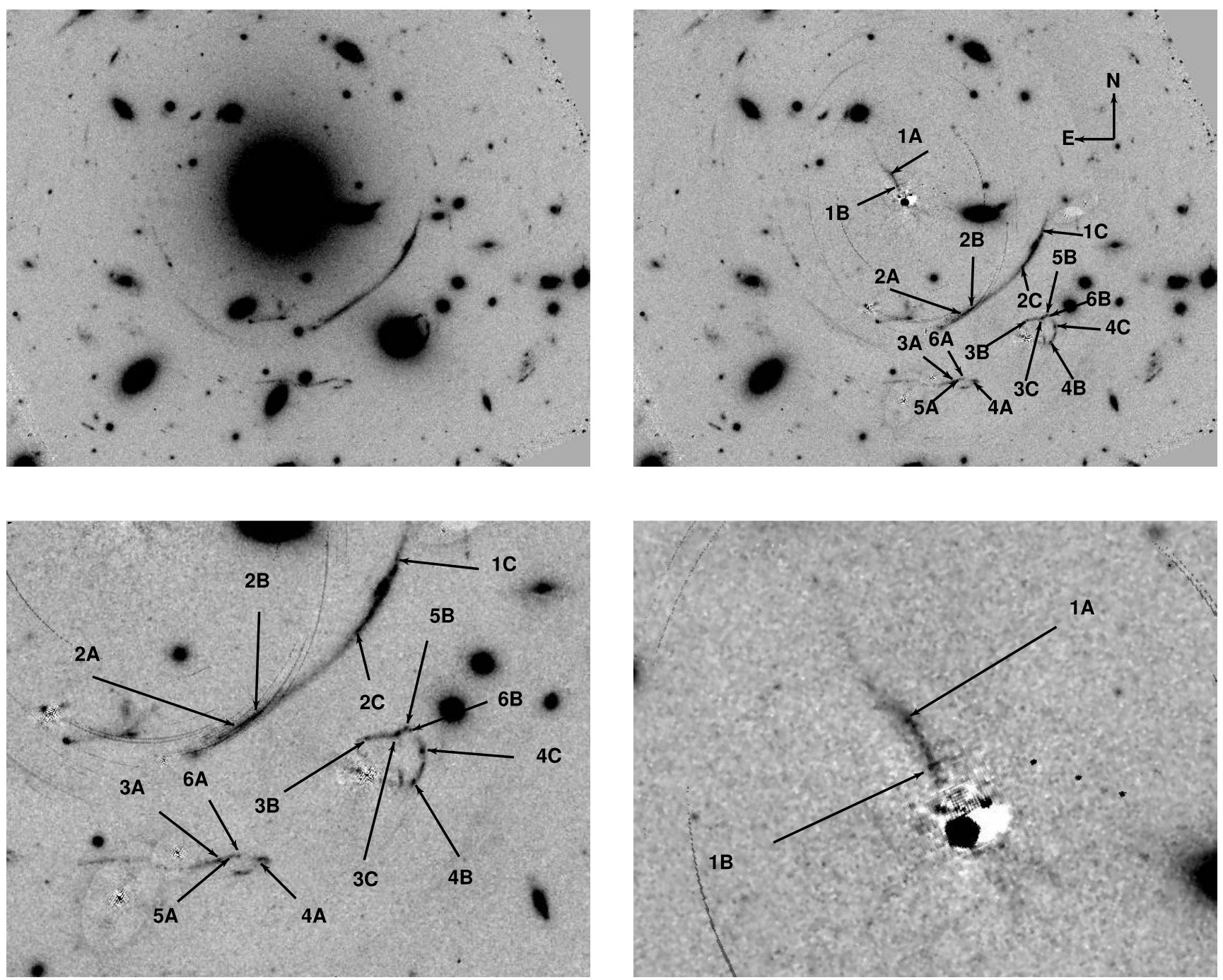

FIG. 2.-Multiple-image interpretation of the cluster Abell 383. The exact positions used are shown in Table 3. In all panels except for the top left we have subtracted cluster galaxies in order to more clearly see the multiple-image features.

those related to multiple-image sets $3,4,5$, and 6 do not have known redshift information. The velocity dispersion data provide three additional constraints. Thus, the resulting number of degrees of freedom is 14 .

\subsubsection{The Fine Positional Accuracy Lensing Case}

The top panels of Figure 4 and the appropriate line in Table 1 summarize the results in this case. DM inner slopes between $\beta=0.5$ and 0.7 lie within the $68 \%$ confidence limit of our analysis (after marginalizing over all other free parameters). The best-fitting scale radius sits again at the edge of the allowed $r_{\mathrm{sc}}$ prior range $\left(r_{\mathrm{sc}}=100 \mathrm{kpc}\right)$. An X-ray analysis of Abell 383, which was able to probe to higher radius than the current analysis, indicates that the DM scale radius is well above $100 \mathrm{kpc}$ (Zhang et al. 2007). For these reasons and those discussed earlier, there is no case for adjusting the DM scale radius prior.

The total $\chi^{2}=40.4$, high given the 14 degrees of freedom in the analysis.

\subsubsection{The Coarse Positional Accuracy Lensing Case}

The bottom panels of Figure 4, along with Table 1, summarize the results for the coarse positional accuracy case. DM inner slopes between $\beta=0.2$ and 0.65 lie within our $68 \% \mathrm{CL}$, along with a best-fitting DM scale radius of $100 \mathrm{kpc}$. Our parameter constraints encompass the values found in the fine accuracy case with no shift in parameter space (unlike the case for MS 2137-23). This suggests that although we should expect a lower $\chi^{2}$ due to the increased uncertainties allowed, no significant improvement to the best-fitting velocity dispersion profile should be expected. As we can see in the bottom right panel of Figure 4, the bestfitting velocity dispersion profile is very similar to that obtained in the fine case. The total $\chi^{2}=22$, acceptable given 14 degrees of freedom.

\section{DISCUSSION}

In the previous section we have presented the results of our analysis, which showed that a mass model comprising a stellar component for the BCG following a Jaffe profile together with a gNFW DM cluster halo is able to adequately reproduce the observations for Abell 383 (albeit only for the coarse lensing positional accuracy scenario) but is unable to simultaneously reproduce the observed multiple-image configuration and BCG velocity dispersion profile for MS 2137-23. In the case of Abell 383, the inner DM profile is flatter than $\beta=1$, supporting the earlier work of S04. This indicates that at least some galaxy clusters have inner DM slopes that are shallower than those seen in numerical simulations, but only if the mass parameterization used in the current work is reflective of reality. Further work in this interesting 

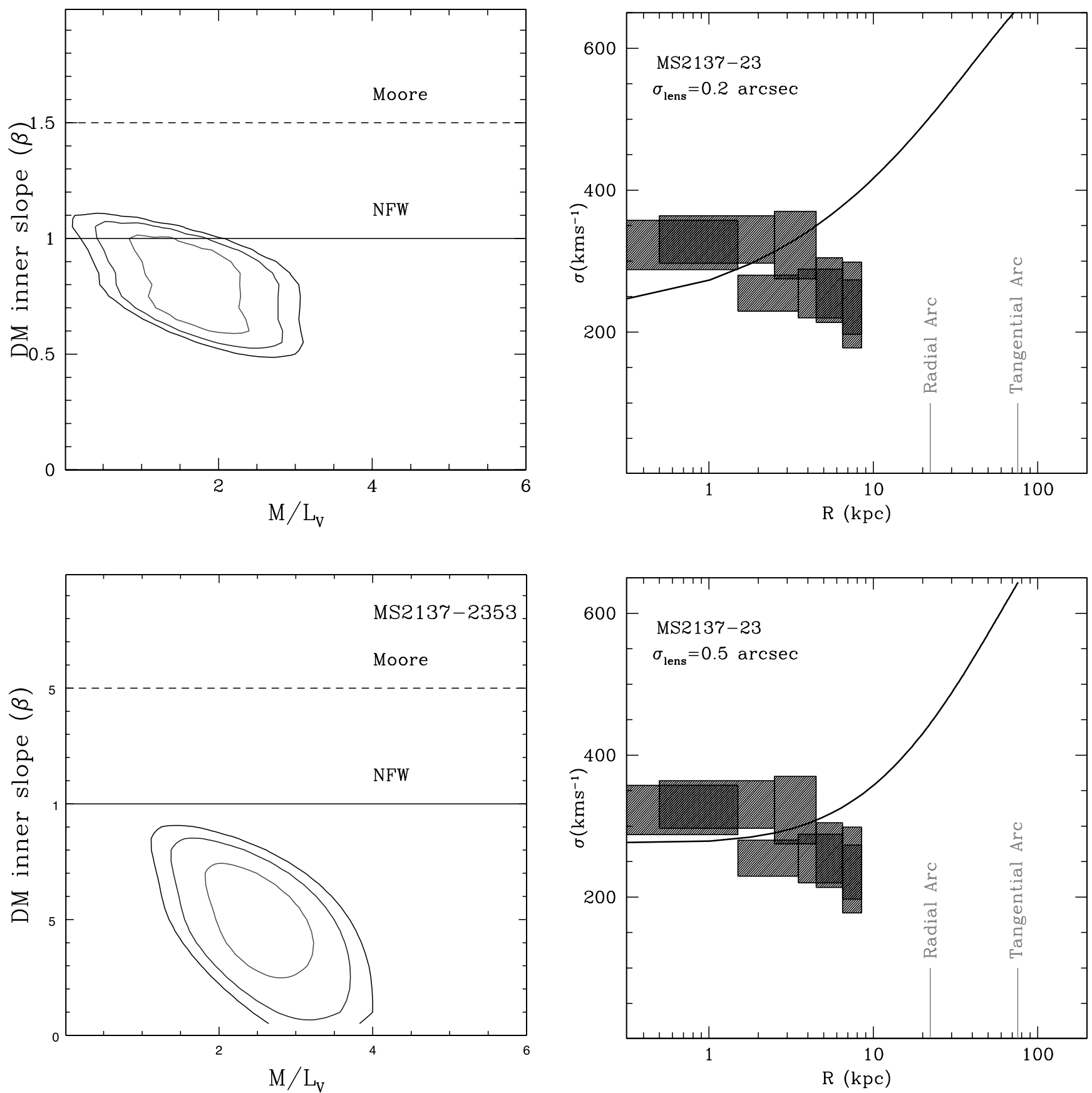

FIG. 3.-Combined lensing+dynamics results for the cluster MS $2137-23$. The top row summarizes the results for the $0.2^{\prime \prime}$ lensing position uncertainty scenario, while the bottom row encapsulates the $0.5^{\prime \prime}$ scenario. Top left: Lensing+dynamics likelihood contours $(68 \%, 95 \%$, and $99 \%)$ in the $M / L-\beta$ plane after marginalizing over the other free parameters with the $0.2^{\prime \prime}$ lensing multiple-image uncertainty. Top right: Best-fitting velocity dispersion profile from the combined lensing + dynamics analysis with the $0.2^{\prime \prime}$ lensing multiple-image uncertainty. No models could be found that fit both the lensing and observed velocity dispersion constraints. Bottom left: Lensing + dynamics likelihood contours $(68 \%, 95 \%$, and $99 \%)$ with the $0.5^{\prime \prime}$ lensing multiple-image uncertainty in the $M / L-\beta$ plane after marginalizing or optimizing over the other free parameters. Bottom right: Best-fitting velocity dispersion profile from the combined lensing + dynamics analysis with the $0.5^{\prime \prime}$ lensing multiple-image uncertainty. While the best-fitting model velocity dispersion is a better fit to the data than in the $0.2^{\prime \prime}$ lensing scenario, it still cannot reproduce the observed decline in the velocity dispersion profile in our highest radial bins, suggesting a problem with our current mass model parameterization. [See the electronic edition of the Journal for a color version of this figure.]

cluster using other observational probes will further refine the mass model and determine if the gNFW DM form is a good fit to the cluster profile.

In this section we discuss systematic uncertainties in our method and possible refinements that could be made to reconcile the mass model with the observations for MS 2137-23. We hope that many of these suggestions will become important as cluster mass models improve and thus will present fruitful avenues of research.

\subsection{Systematic Errors}

We focus first on systematic errors associated particularly with the troublesome stellar velocity dispersion profile for MS 2137-23. Errors could conceivably arise from (1) significant nonGaussianity in the absorption lines (which are fitted by Gaussians), (2) uncertain measurement of the instrumental resolution used to calibrate the velocity dispersion scale, and (3) template mismatch. 

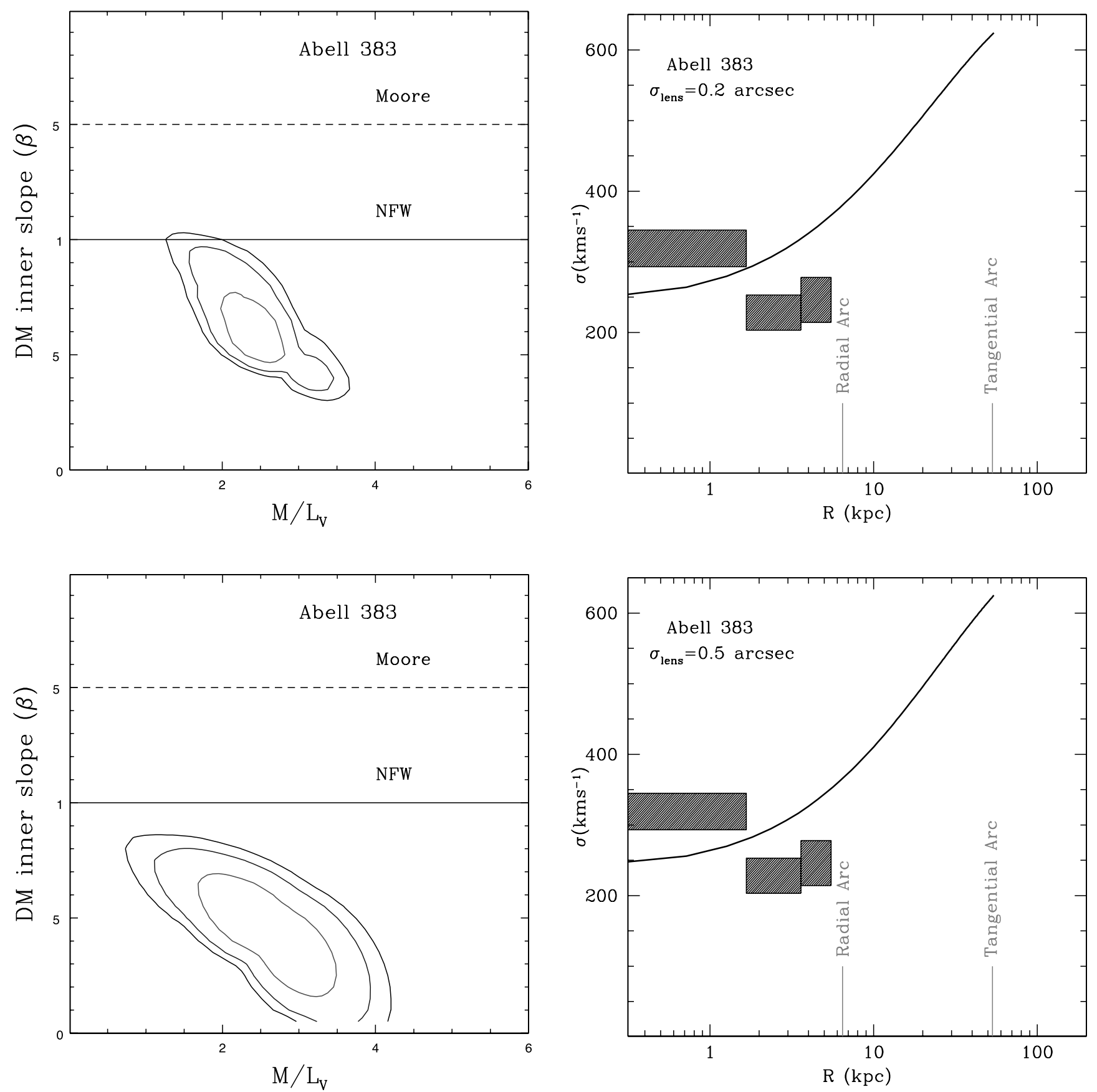

FIG. 4.-Combined lensing+dynamics results for the cluster Abell 383. The top row summarizes the results for the $0.2^{\prime \prime}$ lensing position uncertainty scenario, while the bottom row encapsulates the $0.5^{\prime \prime}$ scenario. Top left: Lensing+dynamics likelihood contours $(68 \%, 95 \%$, and $99 \%)$ in the $M / L-\beta$ plane with the $0.2^{\prime \prime}$ lensing multiple-image uncertainty after marginalizing over the other free parameters. Top right: Best-fitting velocity dispersion profile from the combined lensing + dynamics analysis with the $0.2^{\prime \prime}$ lensing multiple-image uncertainty. Bottom left: Lensing+dynamics contours $(68 \%, 95 \%$, and $99 \%)$ in the $M / L-\beta$ plane with the $0.5^{\prime \prime}$ lensing multiple-image uncertainty after marginalization over the other free parameters. Bottom right: Best-fitting velocity dispersion profile from the combined lensing+dynamics analysis with the $0.5^{\prime \prime}$ lensing multiple-image uncertainty. The $0.5^{\prime \prime}$ lensing multiple-image scenario provides a better overall fit to the observations, although we are limited by the relatively poor quality of the observed Abell 383 velocity dispersion profile. [See the electronic edition of the Journal for a color version of this figure.]

Non-Gaussianity introduces an error that we consider too small to significantly alter the goodness of fit (Gavazzi 2005). The instrumental resolution of ESI (the Keck II instrument used to measure the velocity dispersion profile; Sheinis et al. 2002) is $\sim 30 \mathrm{~km} \mathrm{~s}^{-1}$; this is much smaller than the measured dispersion. Even if the instrumental resolution was in error by a factor of 2, the systematic shift in $\sigma$ would only be $3 \mathrm{~km} \mathrm{~s}^{-1}$ (using eq. [3] in Treu et al. 1999). This would affect all measurements and not reverse the trend with radius.

Concerning template mismatch, S04 estimated a possible systematic shift of up to $15-20 \mathrm{~km} \mathrm{~s}^{-1}$. This could play a role espe- cially as the signal-to-noise ratio $(\mathrm{S} / \mathrm{N})$ diminishes at large radii, where the discrepancy with the model profile is greatest. To test this hypothesis, we added $20 \mathrm{~km} \mathrm{~s}^{-1}$ in quadrature to only those velocity dispersion data points in MS 2137-23 at $R>4 \mathrm{kpc}$ and recalculated the best-fitting $\chi^{2}$ values. The $\chi^{2}$ is reduced from 31 to 28.8 , a modest reduction that fails to explain the poor fit.

Although selectively increasing the error bars on those data points most discrepant with the model is somewhat contrived, our result does highlight the need for high-S/N velocity dispersion measures out to large radii. A high-quality velocity dispersion profile has been measured locally for Abell 2199 to $\sim 20 \mathrm{kpc}$ 
(Kelson et al. 2002). Interestingly, these high-S/N measures display similar trends to those for MS 2137-23 in the overlap regime, i.e., a slightly decreasing profile at $R \lesssim 10 \mathrm{kpc}$. The dip witnessed in MS 2137-23 is thus not a unique feature, although with deeper measurements we might expect to see a rise at larger radii as a result of the shallow DM profile.

A final potential limitation in the dynamical analysis is the assumption of orbital isotropy. Both S04 and Gavazzi (2005) explored the consequences of mild orbital anisotropy, concluding that a possible offset of $\Delta \beta \sim 0.15$ might result. Even including orbital anisotropy into his analysis, Gavazzi (2005) was unable to fit the observed velocity dispersion profile.

Since we determine our lensing $\chi^{2}$ values in the source plane, we checked to make sure that no extra images were seen after remapping our best-fit lensing+velocity dispersion models back to the image plane. No unexpected images were found, although several images that were explicitly not used as constraints were found, such as the mirror image of radial arc image $2 \mathrm{a}$ in MS 2137 and the complex of multiple images associated with $3 \mathrm{abc}, 5 \mathrm{ab}$, and 6ab in Abell 383 (see Figs. 1 and 2). As discussed in $\S \S 3.2$ and 3.3, some of these multiple images were not used as constraints because we could not confidently identify their position due to either galaxy subtraction residuals or blending with other possible multiple-image systems.

We finally comment on the uncertainties assigned to the multipleimage systems for our lens models. We have presented two sets of results in this work, with assigned image positional accuracies of $\sigma_{I}=0.2^{\prime \prime}$ and $0.5^{\prime \prime}$. We find that a variety of lens models are compatible with the $\sigma_{I}=0.2^{\prime \prime}$ case, and only when the velocity dispersion data are included into the analysis do the data fail to be reproduced by the model. Certainly if we were to further increase the positional errors, at some point a good velocity dispersion fit could conceivably be obtained, but we refrain from doing so in the present work.

Increasing the positional uncertainties is only justified if there is evidence that there are significant missing components in the mass models. Further observations that can probe the mass distribution on fine scales to larger radii and higher quality models that can account for phenomena such as adiabatic contraction in the inner regions of galaxy clusters and triaxiality represent the best way to obtain a more precise picture of the cluster mass distribution.

\subsection{Improving the Mass Model}

We now turn our attention to possible inadequacies in the mass model. It is important to stress that the two diagnostics (lensing and dynamics) adopted in this study probe different scales. The lensing data tightly constrain the mass profile at and outside the radial arc $(\sim 20 \mathrm{kpc})$, while the velocity dispersion constrains the mass profile inside $R \lesssim 10 \mathrm{kpc}$. Since multiple images are numerous and their positions can be more precisely measured than velocity dispersion, ${ }^{10}$ they carry more weight in the $\chi^{2}$ statistic than the kinematic points, producing a best overall fitting model (which is lensing dominated) that is a relatively poor fit to the kinematic data. To improve the model, one must admit either that one of the two components of the modeling is incorrect or that the functional form of the mass profile chosen to extrapolate the lensing information at the scales relevant for dynamics is insufficient. In this section we discuss several areas where the mass model presented in this paper could be improved.

\footnotetext{
${ }^{10}$ The error on the astrometry with respect to the relevant scale, the Einstein radius $\theta_{\mathrm{E}}$, is much smaller than the relative error on velocity dispersion, i.e., $\delta \theta / \theta_{\mathrm{E}} \ll \delta \sigma / \sigma$.
}

\subsubsection{The Contribution of the Brightest Cluster Galaxy}

We might query the assumption of a Jaffe density profile for the BCG. This seems an unlikely avenue for improvement given that the Jaffe profile fits the observed BCG surface brightness profile remarkably well (see Fig. 2 of S04). Moreover, Gavazzi (2005) utilized a Hernquist mass profile in his analysis of MS 2137-23, which also matches the observations, and Gavazzi (2005) was likewise unable to reproduce the observed S04 velocity dispersion profile.

We have additionally checked our assumptions by altering the PIEMD fit to the BCG surface brightness data so that it is matched not to the derived Jaffe profile fit to the BCG but directly to the $H S T$ surface brightness profile. With this setup, we found an $r_{\text {cut }}$ value of $23.70 \mathrm{kpc}$ for MS 2137 and $28.65 \mathrm{kpc}$ for Abell 383 (compare this with the numbers in Table 2). Redoing our analysis for the best-fitting $r_{\mathrm{sc}}$ scenario only, our constraints on $\beta$ for both Abell 383 and MS 2137 did not change by more than 0.05 , and so it is not likely that our method for constraining the BCG mass contribution is the root cause of our inability to fit the data to a BCG+gNFW cluster DM halo mass model.

Conceivably the BCG may not be coincident with the center of the cluster DM halo, as has been assumed throughout this work. It is often the case that small subarcsecond offsets between BCGs and cluster DM halos are necessary to fit lensing constraints (e.g., Smith et al. 2005). There is strong evidence that the $\mathrm{BCG}$ is nearly coincident with the general cluster DM halo in projection from the strong-lensing work presented here and by others (Gavazzi et al. 2003; Gavazzi 2005). However, an offset could be responsible for the flat to falling observed velocity dispersion profile if the BCG were actually in a less dark matterdominated portion of the cluster. Another possibility is that there are multiple massive structures along the line of sight, which would be probed by the strong-lensing analysis, but not with the velocity dispersion profile of the BCG. A comprehensive redshift survey of MS 2137-23 could provide further information on structures along the line of sight.

\subsubsection{The Advantage of a Mass Probe at Larger Radii}

With our presented data set, we have seen that it is difficult to constrain the DM scale radius, $r_{\mathrm{sc}}$, because both of our mass probes are only effective within the central $\sim 100 \mathrm{kpc}$ of the clusters, within the typical DM scale radius observed and seen in CDM simulations. For this reason, the inferred DM scale radius for both Abell 383 and MS 2137-23 lay at the boundary of our assumed prior range. Future work will benefit from weak-lensing data, along with galaxy kinematics and X-ray data of the hot ICM that can each probe out to large clustercentric radii.

Although not the focus of the current work, pinning down the correct DM scale radius will be crucial for constraining other DM mass parameters. For instance, there is a well-known degeneracy between $r_{\mathrm{sc}}$ and the inner slope $\beta$ (e.g., Gavazzi et al. 2003; Gavazzi 2005). To briefly explore this, we have rerun our analysis (for the coarse positioning lensing case) for both clusters with an $r_{\mathrm{sc}}$ of 50 and $400 \mathrm{kpc}$, factors of 2 beyond our chosen $r_{\text {sc }}$ prior. We show our confidence contours in Figure 5, which are noteworthy. For example, in the case of MS 2137-23, if we fix $r_{\mathrm{sc}}=50 \mathrm{kpc}$, then the best-fitting $\beta=0.05$. However, if $r_{\mathrm{sc}}=$ $400 \mathrm{kpc}$, then $\beta=0.7$, more in accordance with simulations. Interestingly, the $r_{\mathrm{sc}}=400 \mathrm{kpc}$ scenario returns a better overall $\chi^{2} \sim 26$ than any model with $r_{\mathrm{sc}}=100-200 \mathrm{kpc}$, even though an $r_{\mathrm{sc}}$ of $400 \mathrm{kpc}$ is clearly ruled out by extant weak-lensing observations. None of the other $r_{\mathrm{sc}}=50$ and $400 \mathrm{kpc}$ scenarios produced $\chi^{2}$ values that were comparable to those seen with 

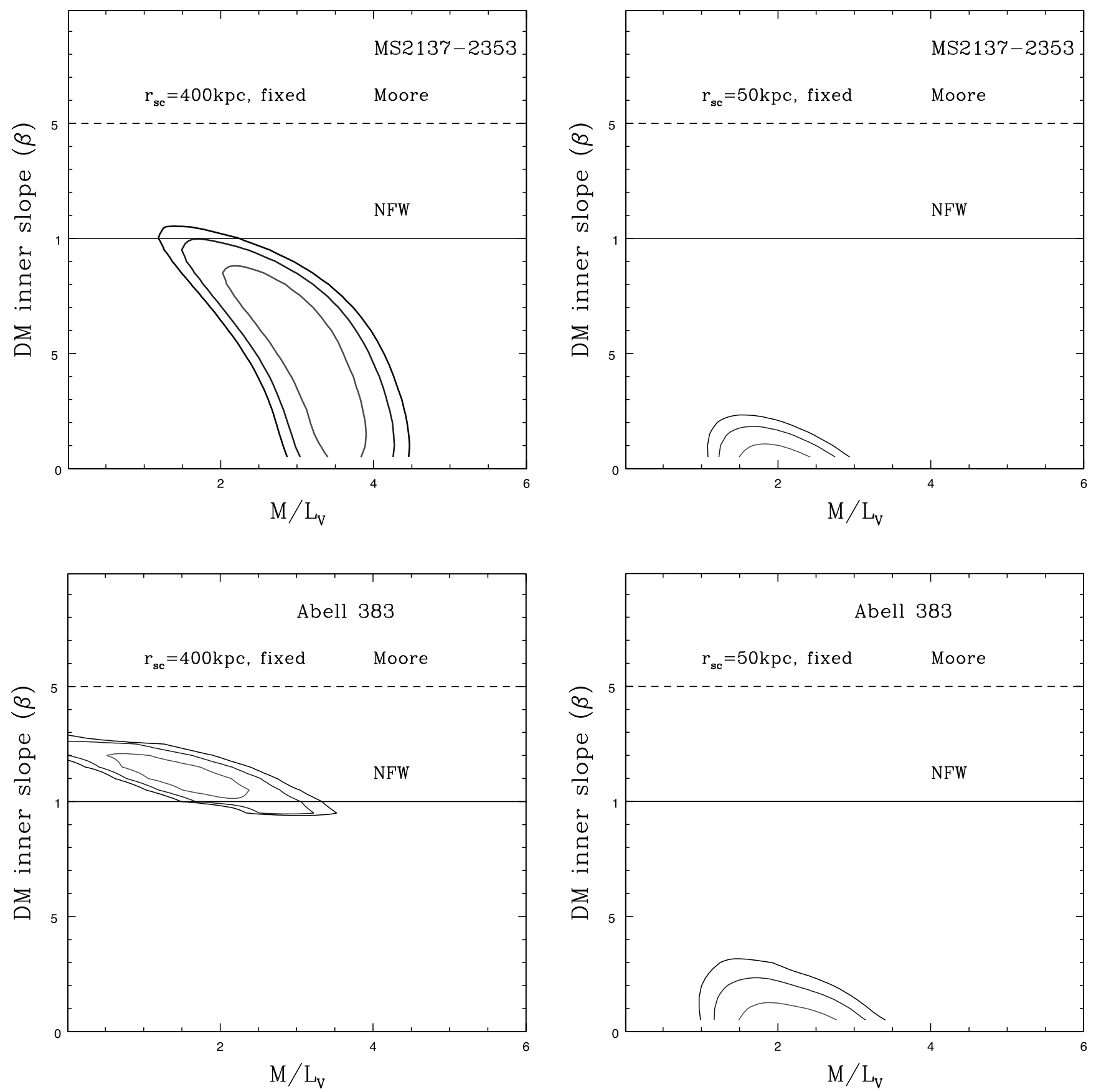

FIG. 5.-Confidence contours $(68 \%, 95 \%$, and $99 \%)$ when we allow the dark matter scale radius to be fixed at values a factor of 2 beyond our observationally motivated prior. Top: Contours when we fix the dark matter scale radius to $r_{\mathrm{sc}}=50$ and $400 \mathrm{kpc}$ in MS 2137. Although the $r_{\mathrm{sc}}=400 \mathrm{kpc}$ scenario provides a relatively good fit to the data $\left(\chi^{2} \sim 26\right)$, this value for the scale radius is much larger than that observed from weak-lensing data. The $r_{\mathrm{sc}}=50 \mathrm{kpc}$ scenario is a significantly worse fit to the data, with $\chi^{2} \sim 39$. Note that the DM inner slope is $\beta<1$ in both scenarios. Bottom: Contours when we fix the dark matter scale radius to $r_{\mathrm{sc}}=50$ and $400 \mathrm{kpc}$ in Abell 383 . The large discrepancy in inner slope values obtained emphasizes the need for a mass probe at larger radii. The best-fitting model for either fixed scale radius is significantly worse than the best-fitting $r_{\mathrm{sc}}=100 \mathrm{kpc}$ result ( $\chi^{2} \sim 26.5$ and 31.3 for $r_{\mathrm{sc}}=50$ and $400 \mathrm{kpc}$, respectively). [See the electronic edition of the Journal for a color version of this figure.]

$r_{\mathrm{sc}}=100-200 \mathrm{kpc}$. Any further knowledge of the DM scale radius would aid greatly in constraining $\beta$ and determining the overall goodness of fit of the gNFW DM profile to the cluster data.

$\mathrm{X}$-ray studies assuming hydrostatic equilibrium (Allen et al. 2001; Schmidt \& Allen 2007) and a combined strong- and weak-lensing analysis (Gavazzi 2005) have presented data on MS 2137-23 to radii much larger than that probed in this study. To check that the mass model derived from data within $\sim 100 \mathrm{kpc}$ does not lead to results at variance with published data at larger radii, we have taken the Gavazzi (2005) results and compared their derived mass at large radii with an extrapolation of our mass models.

Examining Figure 3 from Gavazzi (2005), we estimate a 2D projected mass enclosed from his weak-lensing analysis between $1.6 \times 10^{14}$ and $1.1 \times 10^{15} M_{\odot}$ at $\sim 1.08 \mathrm{Mpc}$ using the cosmology adopted in this paper. Correspondingly, if we take all of the $\Delta \chi^{2}<1.0$ models using our analysis method (the coarse positional accuracy case was used) and calculate the expected 2D projected mass enclosed at $1.08 \mathrm{Mpc}$, we find values between $6.9 \times$ $10^{14}$ and $8.4 \times 10^{14} M_{\odot}$, well within the expected range. 
Note that no attempt was made to extrapolate the mass profiles derived in our analysis to larger radii than the data in this paper allow, although we are acquiring weak-lensing data for a large sample of galaxy clusters to perform a more extensive analysis. The purpose of this consistency check is only to ensure that the masses we derive for such large radii are not too discrepant with existing analyses. The consistency check is satisfied and lends some credence to the models.

\subsubsection{Dark Matter Baryon Interactions and Triaxiality}

The central regions of DM halos can be strongly affected by the gravitational interaction with baryons during halo formation. If stars form and condense much earlier than the DM, it is expected that the baryons will adiabatically compress the DM, resulting in a halo that is steeper than that of the original (Blumenthal et al. 1986; Gnedin et al. 2004). Alternatively, dark matter heating through dynamical friction with cluster galaxies can counteract adiabatic contraction, leading to a shallower DM profile (El-Zant et al. 2004; Nipoti et al. 2004). The present work takes into account neither of the above scenarios, and if any baryon-DM interaction greatly changes the cluster density profile, our assumed parameterized gNFW profile may be inappropriate. Recently, Zappacosta et al. (2006) have used X-ray mass measurements in the cluster Abell 2589 to conclude that processes in galaxy cluster formation serve to counteract adiabatic contraction in the cluster environment. Certainly, more observational work is needed to understand the interplay between baryons and DM in clusters, and extended velocity dispersion profiles of BCGs in conjunction with other mass tracers at larger radii could serve as the best testing ground for the interplay of dark and luminous matter.

Not only is there likely significant interplay between baryons and DM in the central regions of clusters, but real galaxy clusters are certainly triaxial and, if ignored, this may lead to biased parameter estimations and discrepancies when combining mass measurement techniques that are a combination of 2D and 3D. Several recent studies have considered the effects of halo triaxiality on observations. Using an $\mathrm{N}$-body hydrodynamical simulation of a disk galaxy and performing a "long-slit" rotation curve observation, Hayashi et al. (2004) found that orientation and triaxial effects can mistake a cuspy DM profile for one that has a constant density core. At the galaxy cluster scale, Clowe et al. (2004) performed mock weak-lensing observations of simulated galaxy clusters and found that the NFW concentration parameter recovered was correlated with the $3 \mathrm{D}$ galaxy cluster orientation. In order to investigate the recent rash of galaxy clusters with observed high concentration parameters in seeming contradiction to the CDM paradigm (Kneib et al. 2003; Gavazzi 2005; Broadhurst et al. 2005a), Oguri et al. (2005) used strong- and weak-lensing data in Abell 1689 along with a set of models that included halo triaxiality and projection effects. Again, it was seen that halo shape causes a bias in mass (and mass profile) determination, although it should be kept in mind that measurements of concentration are extremely difficult (e.g., Halkola et al. 2006), and the recent study of Limousin et al. (2007) has seemed to clear up the concentration parameter controversy for at least Abell 1689.

In terms of the current work, Gavazzi (2005) has pointed out that the inability of his lensing model to fit the MS 2137-23 BCG velocity dispersion profile may be due to halo triaxiality or projected mass along the line of sight (which would increase the mass measured in the lensing analysis but would not be seen in the stellar velocity dispersion). Gavazzi (2005) showed that an idealized prolate halo with an axis ratio of $\sim 0.4$ could explain the velocity dispersion profile in MS 2137-23. Halo triaxiality could also explain the high concentration previously seen in this cluster.
Again, the gap between simulations and observations may be bridged with respect to triaxiality if further steps were taken to compare the two directly. One step in this direction would be the publication of detailed density profiles for the simulations (in 3D or along numerous projected sight lines).

The most recent DM-only simulations have indicated that the standard NFW profile representation of a DM profile (and its Moore et al. [1999] counterpart with an inner slope $\beta \sim 1.5$ ) can be significantly improved by slightly altering the model to a profile with a slope that becomes systematically shallower at small radii (e.g., Navarro et al. 2004; but see Diemand et al. 2005). While we have adopted the traditional gNFW profile in this study, future work with parameterized models should move toward the latest fitting functions along with an implementation of adiabatic contraction as has already been attempted by Zappacosta et al. (2006). Note, however, that both Navarro et al. (2004) and Diemand et al. (2004) have stated that all fitted functions have their weaknesses when describing complicated $N$-body simulations and when possible simulations and observations should be compared directly.

\section{SUMMARY AND FUTURE WORK}

We have performed a joint gravitational lensing and dynamical analysis in the inner regions of the galaxy clusters Abell 383 and MS 2137-23 in order to separate luminous baryonic from dark matter in the cluster core. To achieve this, we implemented a new 2D pseudoelliptical gNFW mass model in an updated version of the LENSTOOL software package. This refinement is a natural progression from our earlier attempts to measure the dark matter density profile (Sand et al. 2002; S04).

For the study, we adopted an observationally motivated scale radius prior of $r_{\mathrm{sc}}=100-200 \mathrm{kpc}$. With strong lensing alone, we find that a range of mass parameters and DM inner slopes are compatible with the multiple-image data, including those with $\beta>1$ as seen in CDM simulations. However, including the BCG kinematic constraints for both systems, the acceptable parameter ranges shrink significantly.

We can summarize the results for the two clusters as follows:

1. For the cluster Abell 383 we have found satisfactory $\mathrm{BCG}^{+}$ gNFW cluster DM models only for our coarse lensing positional accuracy scenario. Assuming that this is reflective of the underlying cluster DM distribution, the dark matter inner slope is found to be $\beta=0.45_{-0.25}^{+0.2}$, supporting our earlier contention that some clusters have inner DM profiles flatter than those predicted in numerical simulations.

2. For MS 2137-23 our model is unable to reproduce the observed BCG velocity dispersion profile and the range of accepted inner slopes therefore depends sensitively on the adopted uncertainties in the mass model. This may suggest an unknown systematic uncertainty in our analysis or that we have adopted an inappropriate mass model. We explore the former in considerable detail, extending the quite extensive discussion of S04. However, no obvious cause can be found. If, as we suspect, the cause lies with our adopted mass model, it points to the need for further work concerning the distribution of dark matter in the central regions of galaxy clusters.

Future modeling efforts should include the effects of triaxiality and the influence of baryons on dark matter. It is also critical to obtain high-S/N extended velocity dispersion measurements of more BCGs out to larger radii so that, in conjunction with other mass measurement techniques, the interplay of baryons and dark matter in cluster cores can be studied with a real sample. Some other future directions are straightforward. For example, the deep multiband ACS imaging now being done with galaxy clusters 
(Broadhurst et al. 2005b) allows for literally hundreds of multiple images to be found, significantly increasing the number of constraints and allowing for nonparametric mass modeling (Diego et al. 2005), a crucial addition in case the currently used parameterized models do not correspond to reality. We are eager to find ways to more directly compare simulations with observations so that clearer conclusions can be drawn over whether or not simulations and observations are compatible. This may involve measuring other properties of the dark matter halo rather than a sole emphasis on the inner slope, such as the concentration parameter, $c$. Simulated observations of numerical simulations, such as that presented recently by Meneghetti et al. (2007), offer a clear way forward in understanding the systematics involved in observational techniques and the kinds of observations required to test the current paradigm for structure formation.
We thank Raphael Gavazzi for numerous stimulating conversations. D. J. S. acknowledges support provided by NASA through Chandra Postdoctoral Fellowship grant PF5-60041. T. T. acknowledges support from the Sloan Foundation through a Sloan Research Fellowship. G. P. S. acknowledges financial support from a Royal Society University Research Fellowship. Finally, the authors wish to recognize and acknowledge the cultural role and reverence that the summit of Mauna Kea has always had within the indigenous Hawaiian community. We are most fortunate to have the opportunity to conduct observations from this mountain. This research has made use of the NASA/IPAC Extragalactic Database (NED), which is operated by the Jet Propulsion Laboratory, California Institute of Technology, under contract with the National Aeronautics and Space Administration.

\section{APPENDIX}

\section{A GENERALIZED NFW IMPLEMENTATION IN LENSTOOL}

Here we briefly discuss the implementation of the pseudoelliptical gNFW profile into the LENSTOOL software package. The interested reader is referred to Kneib (1993) and Smith et al. (2005) for further details about the LENSTOOL software. Some of what follows has been presented by Golse \& Kneib (2002) but is reviewed here for continuity and clarity.

Throughout this section we are using the thin-lens approximation with $r^{2}=R^{2}+z^{2}$ and $\boldsymbol{x}=\left(x_{1}, x_{2}\right)=\boldsymbol{R} / r_{\mathrm{sc}}$. By introducing ellipticity into the potential rather than the surface mass density, we make the lensing calculations more tractable given that the deflection angle is just the gradient of the scaled lensing potential. Using the following coordinate substitution of $x$ by $x_{\epsilon}$,

$$
\begin{gathered}
x_{1 \epsilon}=\sqrt{a_{1}} x_{1}, \\
x_{2 \epsilon}=\sqrt{a_{2}} x_{2}, \\
x_{\epsilon}=\sqrt{x_{1 \epsilon}^{2}+x_{2 \epsilon}^{2}}=\sqrt{a_{1} x_{1}^{2}+a_{2} x_{2}^{2}}, \\
\phi_{\epsilon}=\arctan \left(x_{2} / x_{1}\right),
\end{gathered}
$$

where $a_{1}$ and $a_{2}$ are two elliptical parameters. We can calculate the elliptical deflection angle:

$$
\boldsymbol{\alpha}_{\epsilon}(\boldsymbol{x})=\left[\begin{array}{l}
\frac{\partial \varphi_{\epsilon}}{\partial x_{1}}=\alpha\left(x_{\epsilon}\right) \sqrt{a_{1}} \cos \phi_{\epsilon} \\
\frac{\partial \varphi_{\epsilon}}{\partial x_{2}}=\alpha\left(x_{\epsilon}\right) \sqrt{a_{2}} \sin \phi_{\epsilon}
\end{array}\right] .
$$

The above expression holds for any definition of $a_{1}$ and $a_{2}$, which we choose to be

$$
\begin{aligned}
& a_{1}=1+\epsilon, \\
& a_{2}=1-\epsilon .
\end{aligned}
$$

While this choice of $a_{1}$ and $a_{2}$ does not correspond directly to the ellipticity of the potential (see Meneghetti et al. [2003], who use a different parameterization), it does lead to simple expressions for standard lensing quantities, such as the surface mass density and shear (see eqs. [17]-[19] of Golse \& Kneib 2002). The standard ellipticity of the potential $\epsilon_{\varphi}$ is related to $\epsilon$ by

$$
\epsilon_{\varphi}=1-\sqrt{\frac{1-\epsilon}{1+\epsilon}} .
$$

Using the standard lensing functions (see, e.g., Miralda-Escude 1991) for the deflection angle $(\alpha)$, convergence $(\kappa)$, and shear $(\gamma)$, along with equation (A2), the projected mass density $\Sigma_{\epsilon}(\boldsymbol{x})$ for our pseudoelliptical implementation is simply

$$
\Sigma_{\epsilon}(\boldsymbol{x})=\Sigma\left(\boldsymbol{x}_{\epsilon}\right)+\epsilon \cos 2 \phi_{\epsilon}\left[\bar{\Sigma}\left(\boldsymbol{x}_{\epsilon}\right)-\Sigma\left(\boldsymbol{x}_{\epsilon}\right)\right]
$$

Likewise, the 3D pseudoelliptical density profile can be similarly derived to be

$$
\rho_{\epsilon}(\boldsymbol{x}, z)=\rho\left(\boldsymbol{x}_{\epsilon}, z\right)+\epsilon \cos 2 \phi_{\epsilon}\left[\frac{2}{x_{\epsilon}^{2}} \int_{0}^{x_{\epsilon}} x \rho(\boldsymbol{x}, z) d x-\rho\left(\boldsymbol{x}_{\epsilon}, z\right)\right],
$$




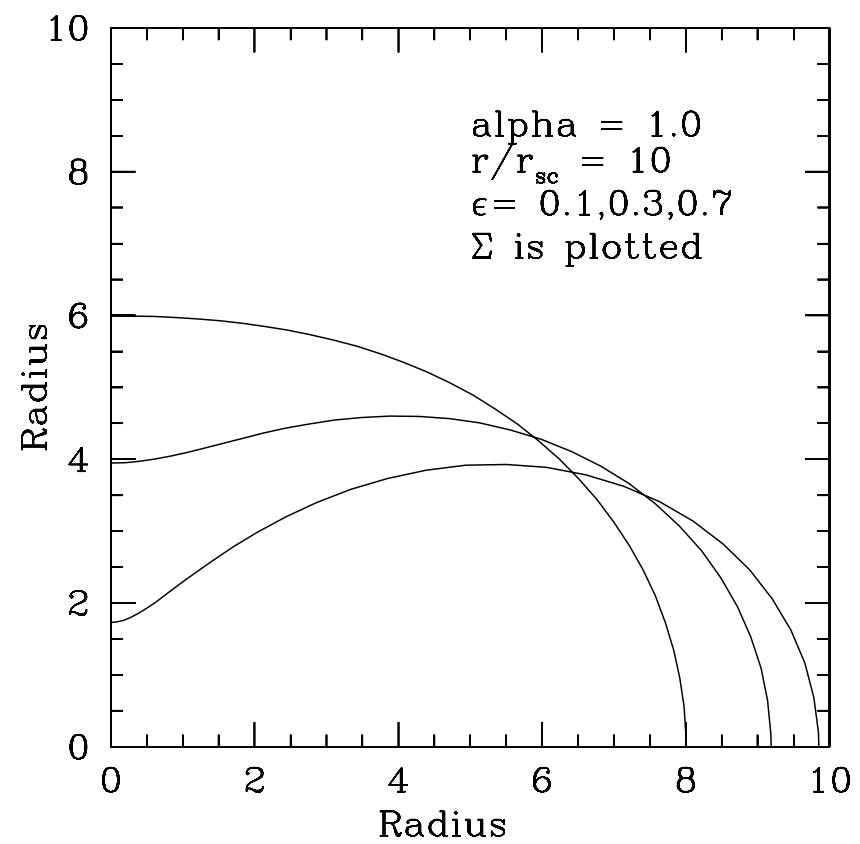

FIG. 6.-Illustration of projected density isocontours for the pseudoelliptical gNFW parameterization with $r / r_{\mathrm{sc}}=10.0$ and $\beta=1.0$. Note that as $\epsilon$ gets larger, the projected density contours become more dumbbell shaped.

where $z$ is the direction along the line of sight. Since we are not making an effort to probe the triaxiality of our galaxy clusters, we plot projected quantities whenever possible.

Although equations (A6) and (A5) are general, we are working with the gNFW density profile

$$
\rho_{d}(r)=\frac{\rho_{c} \delta_{c}}{\left(r / r_{\mathrm{sc}}\right)^{\beta}\left[1+\left(r / r_{\mathrm{sc}}\right)\right]^{3-\beta}}
$$

and the resulting surface density profile

$$
\Sigma_{\mathrm{gNFW}}(R)=2 \rho_{c} r_{\mathrm{sc}} \delta_{c} x^{1-\beta} \int_{0}^{\pi / 2} d \theta \sin \theta(\sin \theta+x)^{\beta-3}
$$

when modeling the cluster dark matter halos.

In Figure 6 the extent to which our implementation does not produce surface density profiles with true elliptical isocontours is illustrated. As the parameter $\epsilon$ increases, the surface density isocontours become more boxy and peanut shaped. However, at relatively low $\epsilon$, the isocontours are very nearly elliptical. We discuss in the following section to what extent our gNFW pseudoelliptical mass model is an adequate description of an elliptical mass distribution.
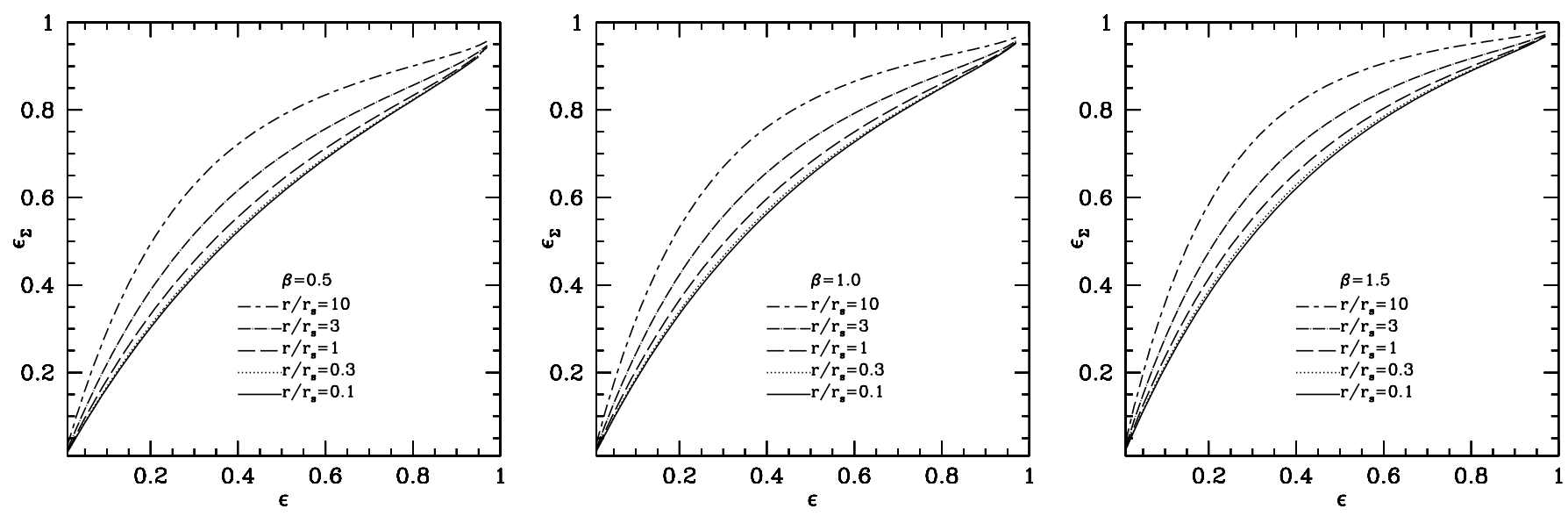

FIG. 7.-Ellipticity (taking the minor- and major-axis positions and assuming $\epsilon_{\Sigma}=1-b / a$ ) of the projected density, $\Sigma$, as a function of the ellipticity in the potential for different values of $\beta$. 

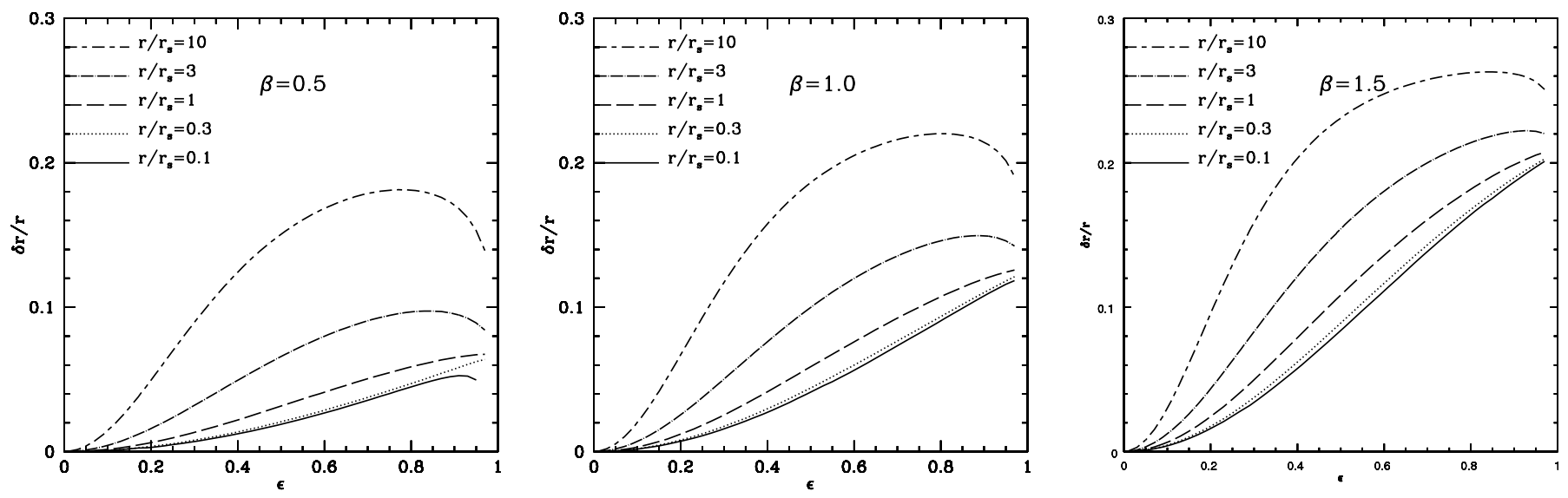

FIG. 8. - Parameter $\delta r / r$ as a function of $\epsilon$ for a variety of pseudoelliptical gNFW models with different inner slopes, $\beta$. This simply characterizes the deviation of the projected density from an elliptical model for various $r / r_{\mathrm{sc}}$

Unlike the NFW profile, the surface mass density and deflection angle of the gNFW profile cannot be calculated analytically. This greatly slows any lensing computation, especially when we need to calculate $\chi^{2}$ values over large parameter hypercubes. To limit the computing time necessary, we created a lookup table for all of the necessary integrals from which we interpolate when performing our lensing calculations.

\section{A1. LIMITATIONS OF THE PSEUDOELLIPTICAL TREATMENT}

In this section we quantitatively investigate the range of $\epsilon$ for which the gNFW pseudoelliptical mass model is an adequate description of an elliptical mass distribution. As can be seen in Figure 6, our pseudoelliptical representation can depart strongly from a true elliptical model at high ellipticities. To what degree can we consider our treatment of ellipticity an accurate one for representing elliptical surface density distributions? To answer this, we reapply several of the quantitative measures presented by Golse \& Kneib (2002) to our gNFW pseudoelliptical model. To get a feel for the relation between $\epsilon$ and the ellipticity in the surface mass density, $\epsilon_{\Sigma}$, we plot several values in Figure 7.

We quantify the degree of boxiness by measuring the distance, $\delta r$, between a surface density contour and a real ellipse with the same major- and minor-axis radii (as was done in Golse \& Kneib 2002; see their Fig. 6 for a geometrical illustration of $\delta r$ ). In Figure 8 we plot $\delta r / r$ as a function of $\epsilon$ for several values of the inner slope, $\beta$, and a variety of $r / r_{\mathrm{sc}}$. If we desire our pseudoelliptical implementation to be within $10 \%$ of a true elliptical surface density distribution for $r / r_{\mathrm{sc}}<10$, then values of $\epsilon \lesssim 0.2$ are appropriate, especially for DM halos with steep inner slopes.

One unphysical consequence of introducing ellipticity into the potential is that the surface mass density can become negative, especially near the minor axis where $\cos \left(2 \phi_{\epsilon}\right)=-1$. In Figure 9 we plot the distance along the minor axis at which $\Sigma_{\epsilon}$ becomes

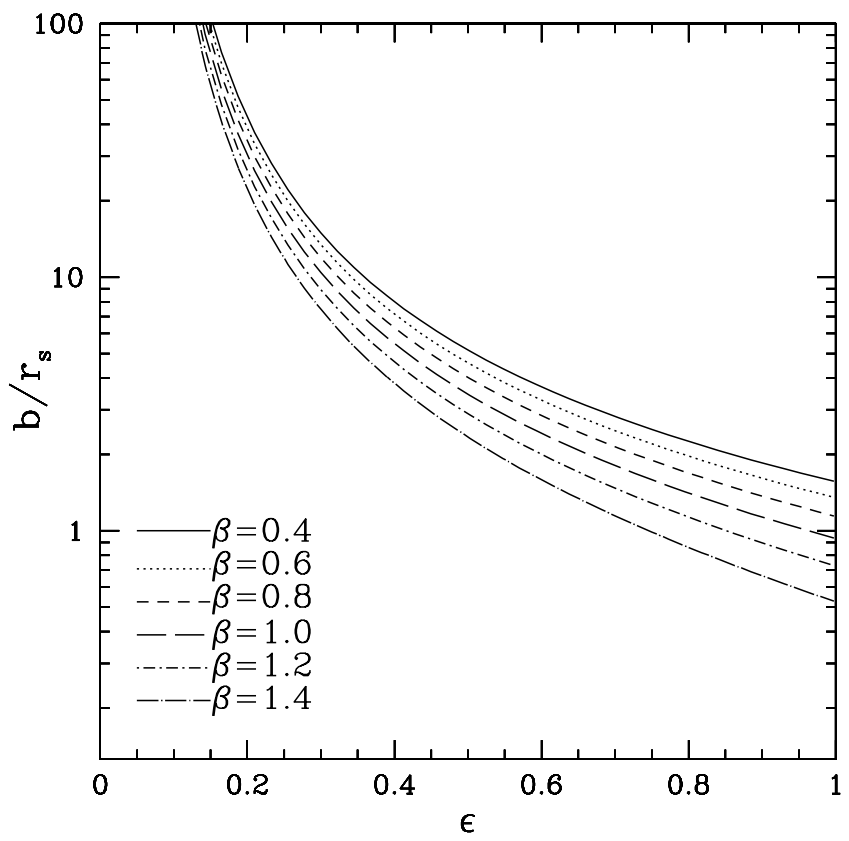

FIG. 9.-Distance from ellipse center along the minor axis at which $\Sigma_{\epsilon}$ becomes negative. Several example values for different inner dark matter density slopes, $\beta$, are plotted. 
negative for several inner slopes. If we wish to restrict ourselves to values of $\epsilon$ where the surface density does not go negative for $r / r_{\mathrm{sc}}<10$, then we must restrict ourselves to values of $\epsilon$ less than approximately 0.25 .

In summary, in order to be within $10 \%$ of a true elliptical surface mass distribution and to have positive values of the surface mass density for $r / r_{\mathrm{sc}}<10$, we must restrict our use of the pseudoelliptical gNFW parameterization to values of $\epsilon \lesssim 0.2$, well within the model values of $\epsilon$ for the clusters studied in this paper.

\section{REFERENCES}

Allen, S. W., Schmidt, R. W., \& Fabian, A. C. 2001, MNRAS, 328, L37

Bartelmann, M., \& Meneghetti, M. 2004, A\&A, 418, 413

Biviano, A., \& Salucci, P. 2006, A\&A, 452, 75

Blumenthal, G. R., Faber, S. M., Flores, R., \& Primack, J. R. 1986, ApJ, 301, 27

Bradač, M., et al. 2006, ApJ, 652, 937

Broadhurst, T., Takada, M., Umetsu, K., Kong, X., Arimoto, N., Chiba, M., \& Futamase, T. 2005a, ApJ, 619, L143

Broadhurst, T., et al. 2005b, ApJ, 621, 53

Bullock, J. S., Kolatt, T. S., Sigad, Y., Somerville, R. S., Kravtsov, A. V.,

Klypin, A. A., Primack, J. R., \& Dekel, A. 2001, MNRAS, 321, 559

Clowe, D., De Lucia, G., \& King, L. 2004, MNRAS, 350, 1038

Dalal, N., \& Keeton, C. R. 2003, preprint (astro-ph/0312072)

Diego, J. M., Sandvik, H. B., Protopapas, P., Tegmark, M., Benitez, N., \& Broadhurst, T. 2005, MNRAS, 362, 1247

Diemand, J., Moore, B., \& Stadel, J. 2004, MNRAS, 353, 624

Diemand, J., Zemp, M., Moore, B., Stadel, J., \& Carollo, C. M. 2005, MNRAS, 364,665

Dolag, K., Bartelmann, M., Perrotta, F., Baccigalupi, C., Moscardini, L., Meneghetti, M., \& Tormen, G. 2004, A\&A, 416, 853

El-Zant, A. A., Hoffman, Y., Primack, J., Combes, F., \& Shlosman, I. 2004, ApJ, 607, L75

Faltenbacher, A., Kravtsov, A. V., Nagai, D., \& Gottlöber, S. 2005, MNRAS, 358,139

Gavazzi, R. 2005, A\&A, 443, 793

Gavazzi, R., Fort, B., Mellier, Y., Pelló, R., \& Dantel-Fort, M. 2003, A\&A, 403,11

Ghigna, S., Moore, B., Governato, F., Lake, G., Quinn, T., \& Stadel, J. 2000, ApJ, 544, 616

Gnedin, O. Y., Kravtsov, A. V., Klypin, A. A., \& Nagai, D. 2004, ApJ, 616, 16

Golse, G., \& Kneib, J.-P. 2002, A\&A, 390, 821

Halkola, A., Seitz, S., \& Pannella, M. 2006, MNRAS, 372, 1425

Hammer, F., Gioia, I. M., Shaya, E. J., Teyssandier, P., Le Fevre, O., \& Luppino, G. A. 1997, ApJ, 491, 477

Hayashi, E., et al. 2004, MNRAS, 355, 794

Jaffe, W. 1983, MNRAS, 202, 995

Kassiola, A., \& Kovner, I. 1993, ApJ, 417, 450

Kelson, D. D., Zabludoff, A. I., Williams, K. A., Trager, S. C., Mulchaey, J. S., \& Bolte, M. 2002, ApJ, 576, 720

Kneib, J., et al. 2003, ApJ, 598, 804

Kneib, J.-P. 1993, Ph.D. thesis, Univ. Paul Sabatier, Toulouse

Kneib, J.-P., Ellis, R. S., Smail, I., Couch, W. J., \& Sharples, R. M. 1996, ApJ, 471,643
Kronawitter, A., Saglia, R. P., Gerhard, O., \& Bender, R. 2000, A\&AS, 144, 53

Limousin, M., Kneib, J.-P., \& Natarajan, P. 2005, MNRAS, 356, 309

Limousin, M., et al. 2007, ApJ, 668, 643

Łokas, E. L., \& Mamon, G. A. 2003, MNRAS, 343, 401

Mahdavi, A., Hoekstra, H., Babul, A., Sievers, J., Myers, S. T., \& Henry, J. P. 2007, ApJ, 664, 162

Mellier, Y., Fort, B., \& Kneib, J.-P. 1993, ApJ, 407, 33

Meneghetti, M., Bartelmann, M., Jenkins, A., \& Frenk, C. 2007, MNRAS, 381, 171

Meneghetti, M., Bartelmann, M., \& Moscardini, L. 2003, MNRAS, 340, 105 Miralda-Escude, J. 1991, ApJ, 370, 1 . 1995, ApJ, 438, 514

Moore, B., Governato, F., Quinn, T., Stadel, J., \& Lake, G. 1998, ApJ, 499, L5

Moore, B., Quinn, T., Governato, F., Stadel, J., \& Lake, G. 1999, MNRAS, 310,1147

Nagai, D., \& Kravtsov, A. V. 2005, ApJ, 618, 557

Natarajan, P., \& Kneib, J.-P. 1997, MNRAS, 287, 833

Navarro, J. F., Frenk, C. S., \& White, S. D. M. 1997, ApJ, 490, 493

Navarro, J. F., et al. 2004, MNRAS, 349, 1039

Nipoti, C., Treu, T., Ciotti, L., \& Stiavelli, M. 2004, MNRAS, 355, 1119

Oguri, M., Takada, M., Umetsu, K., \& Broadhurst, T. 2005, ApJ, 632, 841

Power, C., Navarro, J. F., Jenkins, A., Frenk, C. S., White, S. D. M., Springel, V., Stadel, J., \& Quinn, T. 2003, MNRAS, 338, 14

Sand, D. J., Treu, T., \& Ellis, R. S. 2002, ApJ, 574, L129

Sand, D. J., Treu, T., Smith, G. P., \& Ellis, R. S. 2004, ApJ, 604, 88 (S04)

Schmidt, R. W., \& Allen, S. W. 2007, MNRAS, 379, 209

Sheinis, A. I., Bolte, M., Epps, H. W., Kibrick, R. I., Miller, J. S., Radovan, M. V., Bigelow, B. C., \& Sutin, B. M. 2002, PASP, 114, 851

Smith, G. P., Kneib, J., Ebeling, H., Czoske, O., \& Smail, I. 2001, ApJ, 552, 493

Smith, G. P., Kneib, J.-P., Smail, I., Mazzotta, P., Ebeling, H., \& Czoske, O. 2005, MNRAS, 359, 417

Tasitsiomi, A., Kravtsov, A. V., Gottlöber, S., \& Klypin, A. A. 2004, ApJ, 607, 125

Treu, T., Stiavelli, M., Casertano, S., Møller, P., \& Bertin, G. 1999, MNRAS, 308, 1037

Zappacosta, L., Buote, D. A., Gastaldello, F., Humphrey, P. J., Bullock, J., Brighenti, F., \& Mathews, W. 2006, ApJ, 650, 777

Zentner, A. R., Berlind, A. A., Bullock, J. S., Kravtsov, A. V., \& Wechsler, R. H. 2005, ApJ, 624, 505

Zhang, Y.-Y., Finoguenov, A., Böhringer, H., Kneib, J.-P., Smith, G. P., Czoske, O., \& Soucail, G. 2007, A\&A, 467, 437 